\title{
Automated spectroscopic abundances of A and F-type stars using echelle spectrographs
}

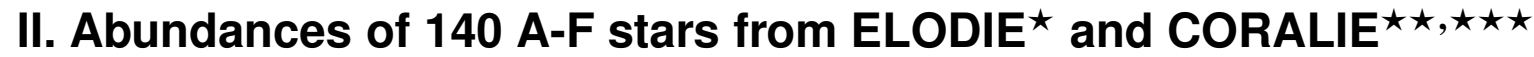

\author{
D. Erspamer and P. North
}

Institut d'astronomie de l'Université de Lausanne, 1290 Chavannes-des-Bois, Switzerland

Received 14 June 2002 / Accepted 26 August 2002

\begin{abstract}
Using the method presented in Erspamer \& North (2002, hereafter Paper I), detailed abundances of 140 stars are presented. The uncertainties characteristic of this method are presented and discussed. In particular, we show that for a $S / N$ ratio higher than 200, the method is applicable to stars with a rotational velocity as high as $200 \mathrm{~km} \mathrm{~s}^{-1}$. There is no correlation between abundances and $V \sin i$, except a spurious one for $\mathrm{Sr}, \mathrm{Sc}$ and $\mathrm{Na}$ which we explain by the small number of lines of these elements combined with a locally biased continuum. Metallic giants (Hauck 1986) show larger abundances than normal giants for at least 8 elements: $\mathrm{Al}, \mathrm{Ca}, \mathrm{Ti}, \mathrm{Cr}, \mathrm{Mn}, \mathrm{Fe}, \mathrm{Ni}$ and $\mathrm{Ba}$. The anticorrelation for $\mathrm{Na}, \mathrm{Mg}, \mathrm{Si}, \mathrm{Ca}, \mathrm{Fe}$ and $\mathrm{Ni}$ with $V \sin i$ suggested by Varenne \& Monier (1999) is not confirmed. The predictions of the Montréal models (e.g. Richard et al. 2001) are not fulfilled in general. However, a correlation between $\left[\frac{\mathrm{Fe}}{\mathrm{H}}\right]$ and $\log g$ is found for stars of 1.8 to $2.0 M_{\odot}$. Various possible causes are discussed, but the physical reality of this correlation seems inescapable.
\end{abstract}

Key words. techniques: spectroscopic - stars: abundances - stars: chemically peculiar - stars: fundamental parameters

\section{Introduction}

A-F type stars present a large number of peculiar types linked with anomalous chemical abundances, such as Ap, Am, $\lambda$ Bootis, $\rho$ Puppis (see Gray \& Garrison 1989b, for a discussion of the $\delta$ Del stars and a definition of the $\rho$ Pup stars). Radiative diffusion is generally invoked to explain most of these peculiarities, and the theory of this mechanism, developed mostly by Michaud and collaborators (Michaud 1991) seems largely successful, even though some adjustments remain necessary. For instance, stellar wind was added to radiative diffusion in order to make more realistic predictions of abundances of Am stars (Michaud et al. 1983).

A little-explored category of chemically peculiar stars in this range of spectral types is that of metallic giants, discovered by Hauck (1986) on the basis of GENEva photometry and further studied by Berthet $(1990,1991)$ using spectroscopy. They are A-F stars leaving the main sequence and having the Geneva

Send offprint requests to: $\mathrm{P}$. North,

e-mail: Pierre.North@obs. unige.ch

* Based on observations collected at the $1.93 \mathrm{~m}$ telescope at the Observatoire de Haute-Provence (St-Michel l'Observatoire, France).

$\star \star$ Based on observations collected at the Swiss $1.2 \mathrm{~m}$ Leonard Euler telescopes at the European Southern Observatory (La Silla, Chile).

$\star \star \star$ Tables 5 and 6 are only available in electronic form at the CDS via anonymous ftp to cdsarc.u.strasbg.fr $(130.79 .128 .5)$ or via http://cdsweb.u-strasbg.fr/cgi-bin/qcat?J/A+A/398/1121 photometric parameter $\Delta m_{2} \geq 0.013$ (see Hauck 1973 for the definition of $\left.\Delta m_{2}\right)$. Berthet $(1990,1991)$ confirmed that these stars have metal abundances higher than solar ones, as suggested by their large $\Delta m_{2}$ values. However, he analyzed only metallic giants and relied on published abundances of normal giants for comparison. Berthet found that the abundance pattern of metallic giants is similar to that of Am stars, except for calcium and scandium, which are notoriously underabundant in the latter, while they are roughly normal or slightly overabundant in the former. On this basis, Berthet (1992) proposed that metallic giants might be evolved Am stars, an idea theoretically justified by the following scenario: in the beginning of the life of an Am star, $\mathrm{Ca}$ and Sc sink and accumulate below the line forming region because of their too small radiative acceleration. During stellar evolution on the main sequence, however, the effective temperature decreases and the outer convection zone develops and thickens, until it reaches the depth where $\mathrm{Ca}$ and $\mathrm{Sc}$ have accumulated and dredges them up, so that their surface abundances becomes normal again. Künzli \& North (1998) challenged this scenario, arguing, in particular, that the $V \sin i$ distribution of metallic giants is not compatible with that of Am stars, especially evolved ones. The fact that metallic giants are more evolved and more massive than normal ones (North et al. 1998) does not fit Berthet's scenario either.

The initial purpose of this work was to focus our attention on metallic giants by comparing their abundances with those 
of normal giants, in order to better define their characteristics and determine how far they form a well defined spectroscopic group. However, since Berthet's scenario remains unsatisfactory, the question of their origin remains open, so we decided to examine these stars in a more general context and try to understand what are their progenitors. So we judged important to study not only giant A-F stars (normal and metallic) but also their possible progenitors, i.e. normal A-F type stars of similar masses on the main sequence. To this purpose, we chose to observe a sample of A-F stars that cover the HR diagram as homogeneously as possible, in order to map the abundance pattern in the whole region of interest. In addition to the homogeneous coverage of the HR diagram, it is important to ensure also the homogeneity of the method of spectral analysis. In the latter sense, homogeneity is crucial, since it is very delicate to compare abundance analyses done by different authors. There is always at least one assumption that differs. The most common problem is the choice of atmospheric parameters, $T_{\text {eff }}$ and $\log g$. Indeed, different techniques, using different photometric systems or spectroscopy, are used to derive atmospheric parameters. Abundance differences arising from these techniques are generally not very important since they remain within error boxes, but they significantly increase the scatter in the results. Another common difference is the choice of atmosphere model theory. In the field of A-F stars, ATLAS9 models from Kurucz (1993) are the rule. However, these models exist with and without overshooting, and both are used depending on the authors. Finally, methods to derive the abundances differ. Some people use equivalent widths while others prefer synthetic spectra adjustments. The oscillator strengths and other line parameters may come from different sources and these sources may vary from one element to the next. The adopted solar iron abundance may also differ from one author to the other. Therefore, even for iron, a large scatter is found. For example, even for a very bright star such as Procyon, $[\mathrm{Fe} / \mathrm{H}]$ values vary from -0.18 to 0.05 (Cayrel de Strobel et al. 2001). All this shows how well justified is the extensive and homogeneous analysis of a large sample of A-F type stars that we present in this paper.

In Sect. 2, we describe the observations and the reduction procedure, while Sect. 3 gives more details on the definition of the sample. The determination of atmospheric parameters is described in Sect. 4, and sources of uncertainties on the derived abundances are discussed in Sect. 5. The results are presented in Sect. 6 and discussed in Sect. 7.

\section{Observations and data reduction}

The spectra used in this work were obtained with two almost identical spectrographs, ELODIE and CORALIE (see Baranne et al. 1996, for technical details about ELODIE and Queloz et al. 2001, for CORALIE). Both spectrographs have been built as a joint project between the Geneva Observatory and the Observatoire de Haute-Provence. ELODIE is attached to the $1.93 \mathrm{~m}$ telescope of the Observatoire de Haute-Provence whereas CORALIE is attached to the Swiss $1.2 \mathrm{~m}$ Leonard Euler telescope at the ESO la Silla Observatory. The optical design of both spectrographs is the same. The major difference is the better resolving power of CORALIE (50000 compared to 42000 for ELODIE) that comes from a slightly different optical combination at the entrance of the spectrograph and the use of a $2 \mathrm{k} \times 2 \mathrm{k}$ CCD camera (against a $1 \mathrm{k} \times 1 \mathrm{k}$ for ELODIE) with smaller pixels (15 $\mu \mathrm{m}$ vs. $24 \mu \mathrm{m})$ (Queloz et al. 1999).

Two observing runs were held in June and August 1999 in Haute-Provence, and one in July 2000 in Chile for a total of 23 nights (10 and 13 respectively). Target stars were bright ones, because we wanted a high signal to noise ratio $(S / N>$ $200)$. The total number of observed stars is 140 , after elimination of all spectroscopic binaries or stars presenting strange cross-correlation functions due e.g. to pulsations.

Data reduction was done as presented in Paper I. As the optical design of CORALIE is the same as the one of ELODIE, only a few changes in the parameters used to characterize the CCD were needed to adapt the IRAF reduction procedure which was initially designed for ELODIE spectra. However, after order extraction, the different orders merge perfectly without scaling, contrary to the case of ELODIE spectra. The whole spectrum is obtained by a simple concatenation of consecutive orders with a detection of the overlapping region. As the $S / N$ ratio is better in the red part of the order than in the blue one, the flux of the first order is retained for $3 / 4$ of the overlapping region, and the flux of the following for the remaining $1 / 4$. The subsequent operations are done using the same procedure as described in Paper I.

\section{Sample}

The mass range is 1.3 to about $2.8 M_{\odot}$, encompassing that of most metallic giants $\left(1.7\right.$ to $\left.3 M_{\odot}\right)$. As we wanted to observe as many stars as possible during the observing runs at our disposal, our choice naturally went to the brighter stars. Two additional requirements were used to define the sample: availability of GenEva photometry (Golay 1980; Rufener \& Nicolet 1988; Cramer 1999) and of HiPPARcos parallaxes (ESA 1997), in order to be able to determine the atmospheric parameters with the method presented in Sect. 4. Known binaries were exluded too. Finally, we chose to keep only stars with $V \sin i<150 \mathrm{~km} \mathrm{~s}^{-1}$ or that have no determination in the bright star catalogue (Hoffleit \& Jaschek 1991). These were the only restrictions before observing.

During the observing runs, as our main targets were metallic giants, we focused a little more on them and on their progenitors, i.e. on stars falling in the mass range 1.7 to $2.5 M_{\odot}$. Low mass stars had been included in the sample essentially because the early Montréal models (see Turcotte et al. 1998; Richer et al. 2000; Richard et al. 2001 and reference therein) did not consider masses larger than $1.5 M_{\odot}$.

As observations were done with spectrographs designed to get radial velocities, the cross-correlation peak was routinely available. We used it to exclude stars presenting a "dubious" peak, i.e. an asymmetric one that may come either from binarity or from pulsation, or a very broad one resulting from $V \sin i$ larger than $200 \mathrm{~km} \mathrm{~s}^{-1}$.

The final sample is composed of 140 stars, out of which 35 are giants (18 metallic and 17 normal), 10 are Am and 6 are Ap. Spectral types are taken from Hauck (1986) for stars in common, and from Gray \& Garrison (1987, 1989a, 1989b) otherwise. The chemically peculiar Am and Ap stars 
are included only because their positions in the HR diagram correspond to the studied region. It is interesting that without taking care of this detail, the proportion of Am and Ap stars, relatively to stars of the main sequence, is representative.

\section{Atmospheric parameters determination}

Atmospheric parameters were determined from Geneva photometry for $T_{\text {eff }}$ and from Hipparcos data (ESA, 1997) for $\log g$. The calib code (Künzli et al. 1997) was used to derive effective temperature from Geneva photometry. Although this code also provides $\log g$, we prefer to determine that value from Hipparcos data. All stars of the sample are bright and have an accurate trigonometric parallax measurement by HIPPARcos. They all have a parallax larger than 7.4 mas (i.e. are closer than $135 \mathrm{pc}$ ) except two, HD 175510 and HD 187764 that are at 166 and $171 \mathrm{pc}$ respectively. Therefore reddening was neglected ${ }^{1}$. Bolometric luminosity was calculated using parallax, bolometric correction interpolated in Flower's table (1996) and $M_{\mathrm{bol}}=4.75$ for the Sun. The bolometric luminosity is then given by

$\log \frac{L}{L_{\odot}}=-0.4\left(M_{v}-4.75+\right.$ B.C. $)$.

The $\log g$ value was then interpolated in the grids of models of Schaller et al. (1992), Schaerer et al. (1993a), Charbonnel et al. (1993) and Schaerer et al. (1993b) according to the method described by North et al. (1997). Finally, an ATLAS9 atmosphere model was computed without overshooting (Castelli et al. 1996) with temperature rounded to $25 \mathrm{~K}$ and $\log g$ to $0.05 \mathrm{dex}$ using the version of ATLAS9 adapted by M. Lemke ${ }^{2}$ for UNIX system.

Derived parameters of our sample stars are presented in Table 5 available at CDS. It contains HD, $T_{\text {eff }} \log g, V_{\text {rad }}, V \sin i$ and $\xi_{\text {micro }}$. The last three values are those derived with our method. Figure 1 shows their position in the HR diagram. Stars cover the whole main sequence and extend slightly beyond.

\section{Uncertainty estimation}

\subsection{Errors resulting from wrong atmospheric parameters}

In order to check the influence of erroneous atmospheric parameters ( $T_{\text {eff }} \pm 150 \mathrm{~K}, \log g \pm 0.2$ dex $)$ on derived abundances,

\footnotetext{
${ }^{1}$ Although this is completely justified for the 121 objects closer than $70 \mathrm{pc}$ on the basis of $u v b y \beta$ photometry, a closer examination shows that the remaining objects are slightly more reddened on average. In particular, 4 stars have $E(b-y)>0.020$ : HD 115604 $(E(b-y)=0.025)$, HD 181333 (0.035), HD 200723 (0.023) and HD 214441 (0.027), implying that $T_{\text {eff }}$ might be underestimated by $\sim 300 \mathrm{~K}$ in the worst case. Among the 121 nearby stars, 8 have also $E(b-y)>0.020$, but they constitute the queue of a fairly Gaussian distribution, 6 of them being closer than $50 \mathrm{pc}$. The colour excesses were estimated using the usual calibrations by Crawford $(1975,1979)$, incorporated in a code kindly made available by Dr. C. Jordi.

2 http://www.sternwarte.uni-erlangen.de/ftp/michael/ atlas-lemke.tgz
}

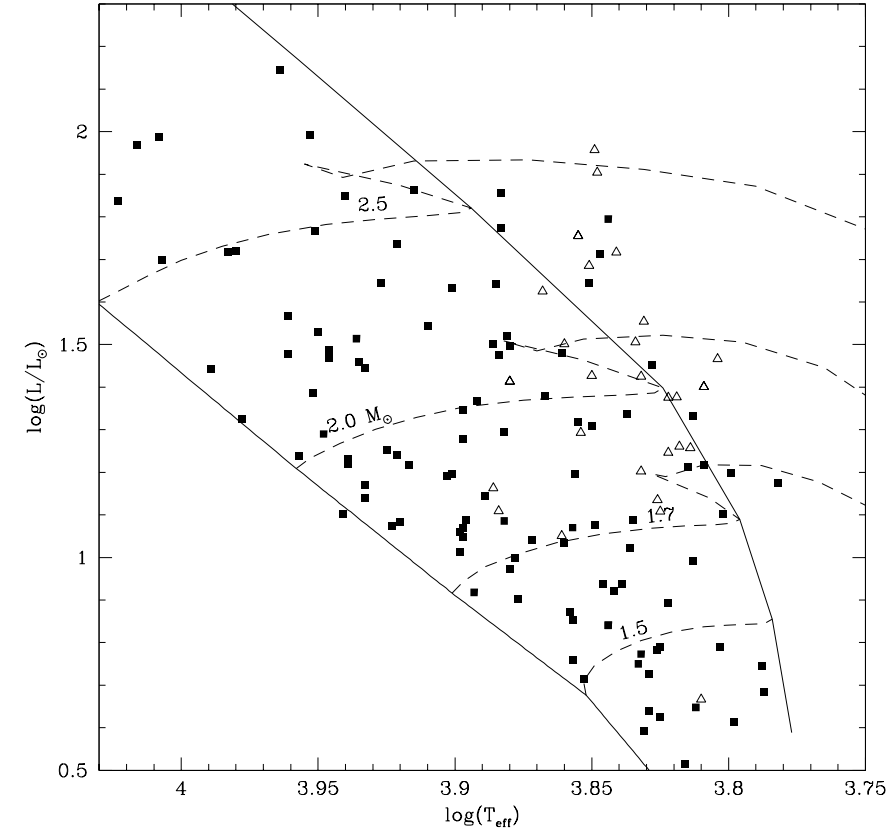

Fig. 1. HR diagram of all studied stars with some evolutionary tracks (Schaller et al. 1992). $\mathbf{m}$ : stars with $\Delta m_{2}<0.013 ; \Delta$ : stars with $\Delta m_{2} \geq$ 0.013 .

simulations were done on a synthetic spectrum. These values do not represent the internal error of the methods used to derive these parameters, but rather the scatter in the estimations done with different methods (photometric or spectroscopic). The synthetic spectrum was computed with a model atmosphere of $T_{\text {eff }}=6950 \mathrm{~K}, \log g=4.0$ and solar chemical composition. This model corresponds to a typical star of our sample lying in the middle of both temperature and gravity ranges. The other parameters, namely radial, microturbulent and especially rotational velocities, are $V_{\text {rad }}=0 \mathrm{~km} \mathrm{~s}^{-1}$, $\xi_{\text {micro }}=1.5 \mathrm{~km} \mathrm{~s}^{-1}$ and $V \sin i=10 \mathrm{~km} \mathrm{~s}^{-1}$. The latter was chosen so that the code returns the input abundances within \pm 0.01 dex when abundances are estimated with the input atmosphere model, the $S / N$ ratio being infinite except for rounding errors. Thus all variations in derived abundances can be attributed to errors on $T_{\text {eff }}$ or $\log g$, even though $V_{\text {rad }}, \xi_{\text {micro }}$ and $V \sin i$ were adjusted, as well as the abundances. The input abundances are solar. The results are presented in Fig. 2 and listed in Table 1.

An erroneous $T_{\text {eff }}$ leads to an error in abundances $\leq 0.1 \mathrm{dex}$ with almost all elements affected in the same way except $\mathrm{C}, \mathrm{O}$, and $\mathrm{S}$ which react in the opposite direction (see Figs. $2 \mathrm{~b}$ and $\mathrm{c}$ ).

It is interesting to note that an erroneous $\log g$ has almost no effect on abundances of iron peak elements ( $\mathrm{Cr}$ to $\mathrm{Zn}$ ), and leads to an error $\leq 0.1 \mathrm{dex}$ for the other elements.

When combining both errors, the total error is always $\leq 0.15 \mathrm{dex}$, as elements that are very sensitive to temperature are not very sensitive to gravity and conversely, at least for the adopted temperature and gravity.

\subsection{Errors resulting from wrong continuum adjustment}

Another source of error comes from bad continuum adjustment. When rotational velocity increases, it becomes harder 
Table 1. Difference between adjusted and reference abundances in hundredth of dex. The last column is the result of the adjustment done with the correct atmosphere model.

\begin{tabular}{|c|c|c|c|c|c|}
\hline Element & $\begin{aligned} & T_{\text {eff }} \\
+ & 150 \mathrm{~K}\end{aligned}$ & $\begin{array}{c}T_{\text {eff }} \\
-150 \mathrm{~K}\end{array}$ & $\begin{array}{c}\log g \\
+0.2 \mathrm{dex}\end{array}$ & $\begin{array}{c}\log g \\
-0.2 \mathrm{dex}\end{array}$ & ref. \\
\hline$V_{\text {rad }}$ & 0 & 0 & 0 & 0 & 0 \\
\hline$V \sin i$ & 0 & 0 & 0 & 0 & 0 \\
\hline$\xi_{\text {micro }}$ & 0 & 0 & 0 & 0 & 0 \\
\hline $\mathrm{C}$ & -3 & 4 & 6 & -6 & -0 \\
\hline $\mathrm{O}$ & -7 & 8 & 7 & -6 & 0 \\
\hline $\mathrm{Na}$ & 6 & -6 & -1 & 1 & -0 \\
\hline $\mathrm{Mg}$ & 5 & -6 & -3 & 3 & -0 \\
\hline $\mathrm{Al}$ & 5 & -6 & -1 & 0 & -1 \\
\hline $\mathrm{Si}$ & 3 & -4 & 0 & -1 & -1 \\
\hline $\mathrm{S}$ & -2 & 2 & 4 & -4 & -1 \\
\hline $\mathrm{Ca}$ & 7 & -8 & -1 & 1 & -0 \\
\hline $\mathrm{Sc}$ & 4 & -5 & 6 & -8 & -1 \\
\hline $\mathrm{Ti}$ & 7 & -7 & 4 & -4 & -0 \\
\hline $\mathrm{V}$ & 7 & -7 & 4 & -4 & -0 \\
\hline $\mathrm{Cr}$ & 6 & -6 & 2 & -2 & -0 \\
\hline $\mathrm{Mn}$ & 8 & -8 & 0 & 0 & 0 \\
\hline $\mathrm{Fe}$ & 8 & -8 & -0 & -0 & -0 \\
\hline $\mathrm{Co}$ & 9 & -8 & 1 & -1 & 0 \\
\hline $\mathrm{Ni}$ & 7 & -9 & -1 & -0 & -1 \\
\hline $\mathrm{Cu}$ & 9 & -10 & -0 & -0 & -0 \\
\hline $\mathrm{Zn}$ & 8 & -8 & 0 & 0 & 0 \\
\hline $\mathrm{Sr}$ & 10 & -9 & 2 & -1 & -0 \\
\hline $\mathrm{Y}$ & 6 & -6 & 7 & -6 & -0 \\
\hline $\mathrm{Zr}$ & 5 & -5 & 7 & -7 & -0 \\
\hline $\mathrm{Ba}$ & 9 & -9 & 3 & -3 & -1 \\
\hline $\mathrm{La}$ & 8 & -5 & 8 & -5 & 1 \\
\hline $\mathrm{Ce}$ & 7 & -7 & 6 & -6 & 0 \\
\hline $\mathrm{Nd}$ & 9 & -8 & 7 & -6 & -0 \\
\hline $\mathrm{Sm}$ & 8 & -7 & 6 & -6 & -0 \\
\hline $\mathrm{Eu}$ & 8 & -8 & 6 & -6 & 0 \\
\hline
\end{tabular}

to find continuum points. The use of echelle spectra is helpful because the whole spectrum from $3900 \AA$ to $6820 \AA$ is at our disposal. The normalization method is presented in Paper I but can be summarized as follows: the spectrum is divided into 6 parts, with one common order between parts, and each part is normalized separately. Each part has a length of more than $400 \AA$ and was chosen so that there are some continuum points near the borders. The continuum is fitted with the continuum function of IRAF whose parameters were defined using slow rotators, mainly the Sun and Vega.

Even when working with high $S / N$ ratios, the main problem for continuum estimation is rotational velocity. It is relatively easy to normalize a spectrum for a star with a $V \sin i \leq$ $40 \mathrm{~km} \mathrm{~s}^{-1}$, because lines are not too broad. When rotational velocity increases, lines become broader and it is hard to find lines that are not blended. Blends become so important that continuum points vanish. Selecting wide parts allows to preserve continuum points even for large rotational velocities.

Simulations were done on synthetic spectra having the same parameters as in previous section, but with $V \sin i=$ $10,50,100,150$ and $200 \mathrm{~km} \mathrm{~s}^{-1}$. Moreover, in order to simulate as closly as possible real spectra, Gaussian noise was added to obtain a reference spectrum with $S / N=200$, which corresponds to the minimum observed value. Finally, the spectra were scaled by a factor 0.99 and 1.01 , so that the continuum level is respectively too high (by a factor 1.01) and too low (by a factor 0.99). For larger differences, it becomes obvious that the continuum is false. Results of these test are presented in Fig. 3 and in Table 2.

It is interesting to note that for the correct continuum definition, almost all adjusted abundances are well within 0.1 dex of their input values, even for high $V \sin i$ (see central column of Fig. 3). Moreover the adjustment with the correct value allows to select "sensitive" elements, such as $\mathrm{Al}, \mathrm{Si}, \mathrm{S}, \mathrm{V}, \mathrm{Cu}$, $\mathrm{Y}$, and $\mathrm{Nd}$. These elements have very few and very weak lines, generally blended with much stronger lines.

Another interesting point is the shape of the abundance pattern obtained with a scaled continuum. This shape remains very constant when the rotational velocity increases, but its amplitude increases with this velocity.

Finally, this simulation can be interpreted as yielding the maximum possible error, because all adjusted parts were scaled by one and the same factor. In reality, even for $V \sin i=$ $150 \mathrm{~km} \mathrm{~s}^{-1}$, only the first two parts $(\lambda<4900 \AA)$ can be affected by the lack of continuum points, the 5 remaining parts being much easier to normalize. Therefore it is possible to conclude that the influence of the normalization on real spectra will be well below the values listed in Table 2 .

\section{Results}

All results are listed in Tables 5 and 6 available at the CDS. The first table lists, for each star, the radial velocity, the microturbulence and the projected rotational velocity. The second one lists the abundances. In 12 cases, there are two spectra per starhence two lines per star in Tables 5 and 6 - either because the same object was observed once with ELODIE and once with CORALIE (6 cases), or because the star was observed twice with a given instrument (6 more cases).

\subsection{Abundances}

As the sample covers a large temperature domain, it is difficult to summarize the results. For early A-type stars, only a few elements show lines in the spectrum while for F-type stars, it is possible to derive abundances for 28 elements. Table 6, available at CDS, contains the detailed abundances for each star and the number of lines used for the determination.

24 stars of the sample are common with the catalogue of $\left[\frac{\mathrm{Fe}}{\mathrm{H}}\right]$ done by Cayrel de Strobel et al. (2001). Comparisons for $T_{\mathrm{eff}}, \log g$ and $\left[\frac{\mathrm{Fe}}{\mathrm{H}}\right]$ are illustrated in Fig. 4. The agreement is very good. It is intersting to notice that 12 stars come from a single, homogeneous source, Balachandran (1990). This sample alone shows a slight shift in $T_{\text {eff }}$ of less than $100 \mathrm{~K}$ and $\log g$ values which are in good agreement with ours. $\left[\frac{\mathrm{Fe}}{\mathrm{H}}\right]$ values are in good agreement, except for two cases where the difference amounts to about 0.3 dex. 


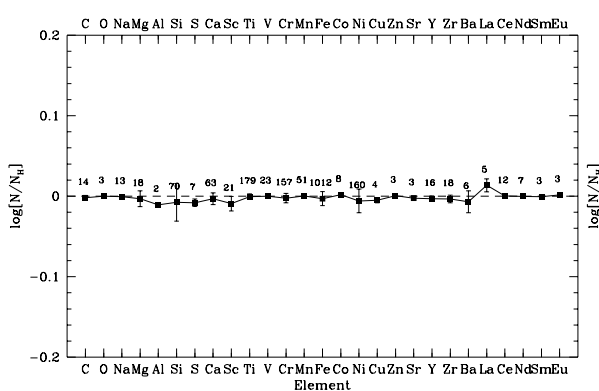

(a) $T_{\text {eff }}=6950 ; \log g=4.0$

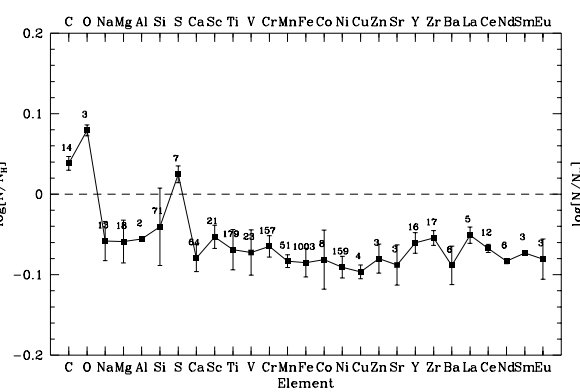

(b) $T_{\text {eff }}=6800 ; \log g=4.0$

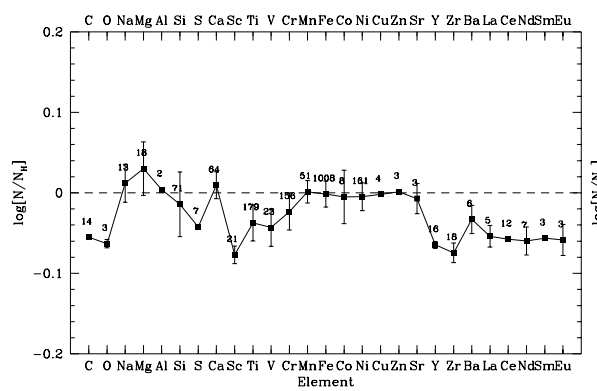

(e) $T_{\text {eff }}=6950 ; \log g=3.8$

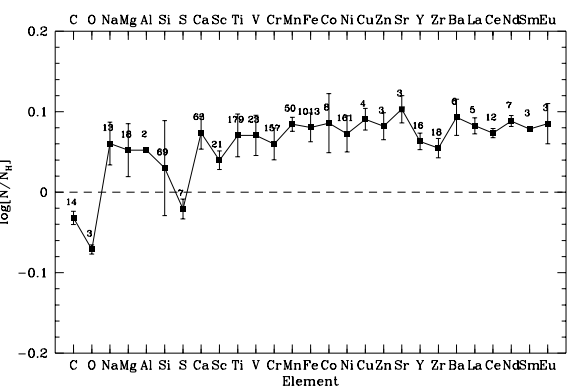

(c) $T_{\text {eff }}=7100 ; \log g=4.0$

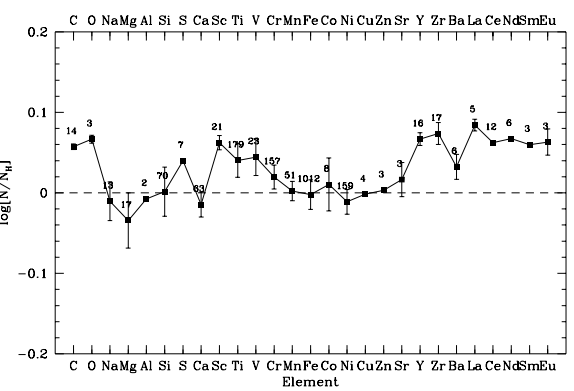

(f) $T_{\text {eff }}=6950 ; \log g=4.2$

Fig. 2. Abundances with respect to the Sun for different values of $T_{\text {eff }}$ and $\log g$. a) Abundances obtained with the correct atmosphere model. b)-e) Abundances obtained with the specified parameters. Error bars represent the sigma of the average abundance value of the 7 parts of the spectrum (see Paper I).

\subsection{Microturbulence}

Microturbulence is an ad hoc parameter which relates to turbulent motions on scales smaller than the mean free path of a photon. As commonly done in practice, we assumed it is constant with optical depth even though it should be allowed to vary in principle. Our estimates are based on the the four bluer spectral segments only, since there are too few appropriate lines in the three redder segments to constrain the adjustment; the microturbulence value is an average of the four individual fitted values. Because of the large size and homogeneity of our sample, the relation between microturbulence, effective temperature and luminosity is worth examining.

The values are reported in Table 5 available at CDS. Figure 5 shows the relation between $T_{\text {eff }}$ and $\xi_{\text {micro }}$ for reliable $\xi_{\text {micro }}$ estimates, defined as those for which the standard deviation of the 4 individual fitted values is smaller than $0.5 \mathrm{~km} \mathrm{~s}^{-1}$. This relation is compared with the average, hand-drawn one presented in Coupry \& Burkhart (1992), which is the continuous curve in Fig. 5. The agreement is good for cool stars but, beyond $T_{\text {eff }}=7400 \mathrm{~K}$, the relation of Coupry \& Burkhart becomes an upper limit to our values.

The relation of Nissen (1981), which is linear in both $T_{\text {eff }}$ and $\log g$, is represented by the three straight lines in its domain of validity. The agreement seems not too bad for low luminosity stars, but our data seem to indicate that all stars share the same $\xi_{\text {micro }}$ at low temperature, whatever their $\log g$, while evolved stars separate from the unevolved ones very rapidly as $T_{\text {eff }}$ increases. The $T_{\text {eff }}$ dependence of $\xi_{\text {micro }}$ is much steeper in our case than in Nissen's, and the slope seems to increase with decreasing $\log g$. Qualitatively, however, we confirm that evolved stars have higher $\xi_{\text {micro }}$ in this range of $T_{\text {eff }}$. This is also in qualitative agreement with the results of Gray et al. (2001) for stars cooler than A8.

Strangely enough, the trend reverses for $T_{\text {eff }}$ between 8000 and $8800 \mathrm{~K}$, evolved stars having significantly lower $\xi_{\text {micro }}$ on average than unevolved ones. The latter trend is largely due to the group of stars with $T_{\text {eff }}$ larger than $8000 \mathrm{~K}$ and $\xi_{\text {micro }}$ larger than 2.4, which correspond to stars with mass of 1.8 to $2.0 M_{\odot}$ that present iron and nickel overabundances (see Sect. 7.3 for details). These stars are very close to the Zero Age Main Sequence (ZAMS) in the HR diagram. This behaviour was already noted by Gray et al. (2001). Whether $\xi_{\text {micro }}$ depends on $[M / H]$ remains an open question, and more high quality data would be needed to better understand what is happening in this diagram. Let us notice that the observed overabundance of iron cannot be the result of a bias in the estimate of $\xi_{\text {micro }}$, since only an underestimate of it would lead to an overestimate of iron abundance; what we see is, on the contrary, rather high $\xi_{\text {micro }}$ values for these metal rich stars.

It is difficult to draw any conclusion about a possible relation between $\xi_{\text {micro }}$ and $\log g$ for the stars hotter than $8800 \mathrm{~K}$ because their number is too small. In addition, the $\xi_{\text {micro }}$ estimates are less reliable than for cool stars because of the much smaller number of lines. 


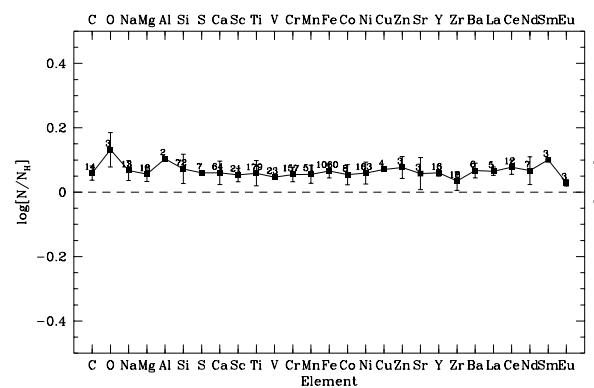

(a) $V \sin i=10 ; \mathrm{S} / \mathrm{N}=200 ;$ cont $=1.01$

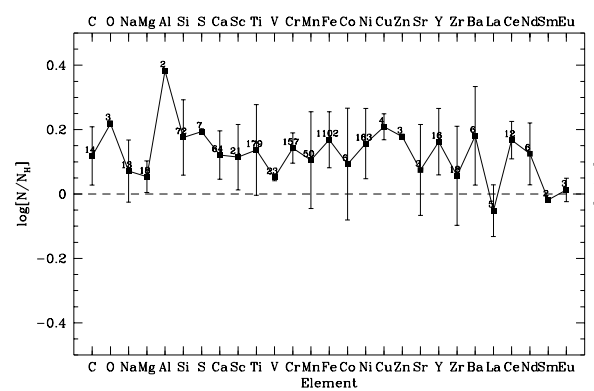

(d) $V \sin i=50 ; \mathrm{S} / \mathrm{N}=200 ;$ cont $=1.01$

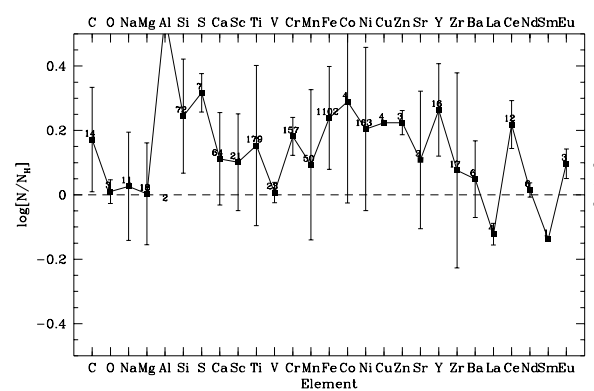

(g) $V \sin i=100 ; \mathrm{S} / \mathrm{N}=200 ;$ cont $=1.01$

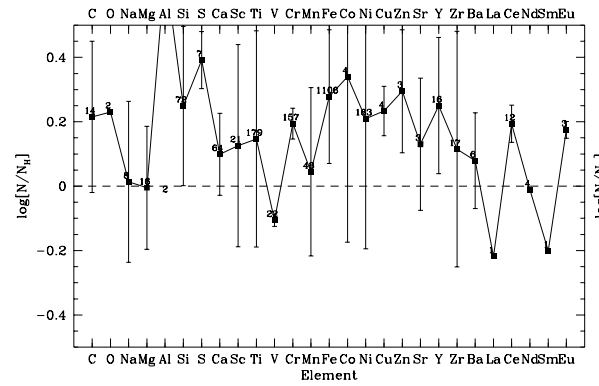

(j) $V \sin i=150 ; \mathrm{S} / \mathrm{N}=200 ;$ cont $=1.01$

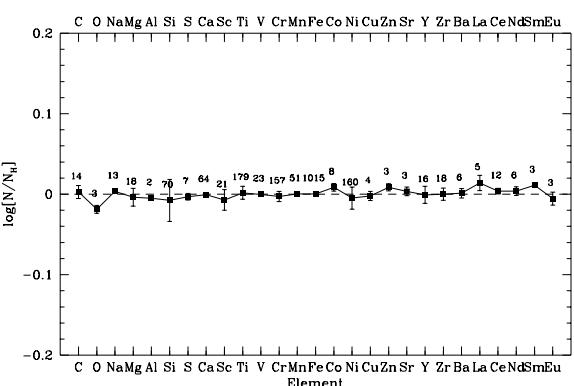

(b) $V \sin i=10 ; \mathrm{S} / \mathrm{N}=200 ;$ cont $=1$

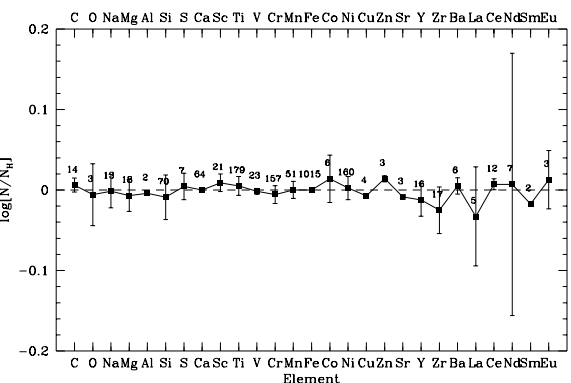

(e) $V \sin i=50 ; \mathrm{S} / \mathrm{N}=200 ;$ cont $=1$

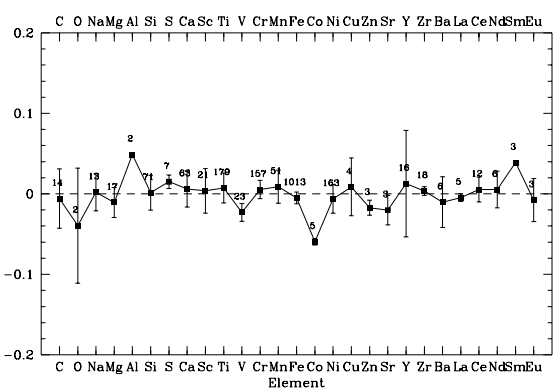

(h) $V \sin i=100 ; \mathrm{S} / \mathrm{N}=200 ;$ cont $=1$

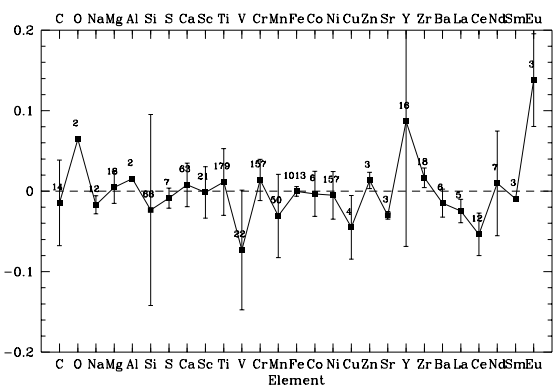

(k) $V \sin i=150 ; \mathrm{S} / \mathrm{N}=200 ;$ cont $=1$

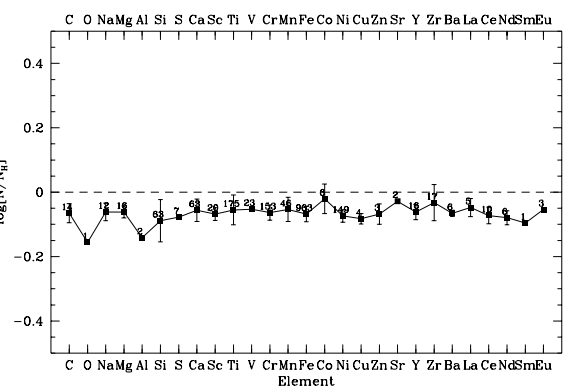

(c) $V \sin i=10 ; \mathrm{S} / \mathrm{N}=200 ;$ cont $=0.99$

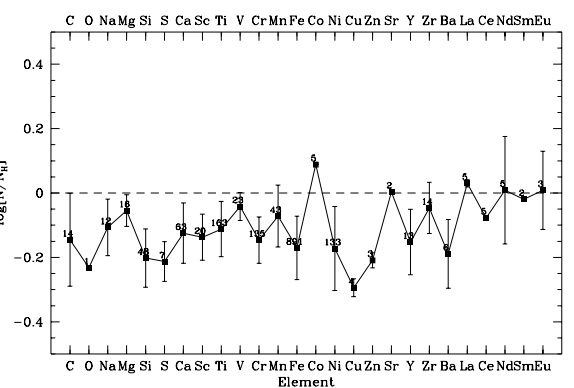

(f) $V \sin i=50 ; \mathrm{S} / \mathrm{N}=200 ;$ cont $=0.99$

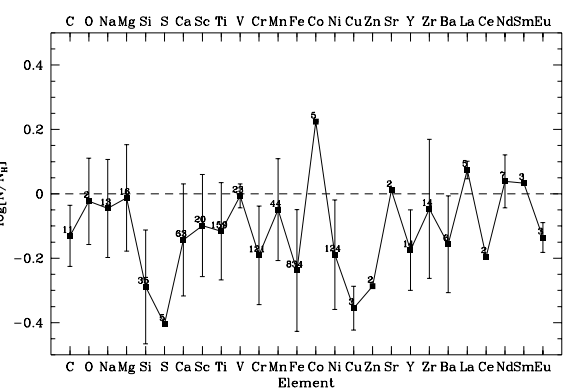

(i) $V \sin i=100 ; \mathrm{S} / \mathrm{N}=200 ;$ cont $=0.99$

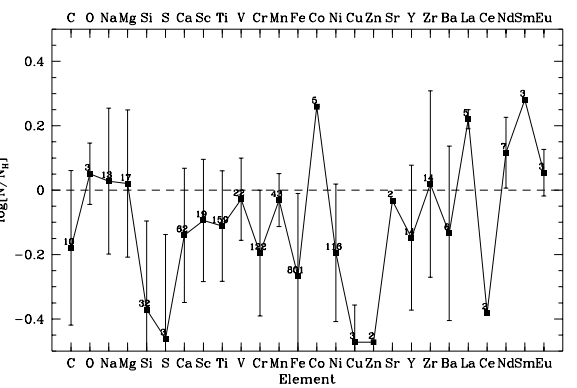

(l) $V \sin i=150 ; \mathrm{S} / \mathrm{N}=200 ;$ cont $=0.99$

Fig. 3. Abundances with respect to the Sun for different continuum heights. Error bars represent the sigma of the average abundance value of the 7 parts of the spectrum (see Paper I). 
Table 2. Difference between adjusted and reference abundances in hundredth of dex for different rotational velocities and different continuum heights.

\begin{tabular}{|c|c|c|c|c|c|c|c|c|c|c|c|c|}
\hline & \multicolumn{3}{|c|}{$\bar{V} V \sin i=10 \mathrm{~km} \mathrm{~s}^{-1}$} & \multicolumn{3}{|c|}{$V \sin i=50 \mathrm{~km} \mathrm{~s}^{-1}$} & \multicolumn{3}{|c|}{$V \sin i=100 \mathrm{~km} \mathrm{~s}^{-1}$} & \multicolumn{3}{|c|}{$V \sin i=150 \mathrm{~km} \mathrm{~s}^{-1}$} \\
\hline & 0.99 & 1. & 1.01 & 0.99 & 1. & 1.01 & 0.99 & 1. & 1.01 & 0.99 & 1. & 1.01 \\
\hline$V_{\mathrm{rad}}$ & 0 & 0 & 0 & 0 & 0 & 0 & 0 & 0 & 0 & 0 & 0 & 0 \\
\hline$V \sin i$ & 0 & 0 & 0 & 0 & 0 & 0 & 0 & 0 & 0 & 0 & 0 & 0 \\
\hline$\xi_{\text {micro }}$ & 0 & 0 & 0 & 0 & 0 & 0 & 0 & 0 & 0 & 0 & 0 & 0 \\
\hline $\mathrm{C}$ & 6 & 0 & -7 & 12 & 1 & -15 & 17 & -1 & -13 & 21 & -1 & -18 \\
\hline $\mathrm{O}$ & 13 & -2 & -16 & 22 & -1 & -23 & 1 & -4 & -2 & 23 & 6 & 5 \\
\hline $\mathrm{Na}$ & 7 & 0 & -6 & 7 & -0 & -11 & 3 & 0 & -5 & 1 & -2 & 3 \\
\hline $\mathrm{Mg}$ & 6 & -0 & -6 & 5 & -1 & -5 & 0 & -1 & -1 & -0 & 0 & 2 \\
\hline $\mathrm{Al}$ & 10 & -0 & -14 & 38 & -0 & & 56 & 5 & & 68 & 2 & \\
\hline $\mathrm{Si}$ & 7 & -1 & -9 & 18 & -1 & -20 & 24 & 0 & -29 & 25 & -2 & -37 \\
\hline $\mathrm{S}$ & 6 & -0 & -8 & 19 & 0 & -21 & 32 & 2 & -40 & 39 & -1 & -46 \\
\hline $\mathrm{Ca}$ & 6 & -0 & -6 & 12 & -0 & -12 & 11 & 1 & -14 & 10 & 1 & -14 \\
\hline $\mathrm{Sc}$ & 5 & -1 & -7 & 11 & 1 & -14 & 10 & 0 & -10 & 13 & -0 & -9 \\
\hline $\mathrm{Ti}$ & 6 & 0 & -6 & 14 & 1 & -11 & 15 & 1 & -12 & 15 & 1 & -11 \\
\hline $\mathrm{V}$ & 5 & -0 & -5 & 5 & -0 & -4 & 1 & -2 & -1 & -11 & -7 & -3 \\
\hline $\mathrm{Cr}$ & 6 & -0 & -6 & 14 & -1 & -15 & 18 & 1 & -19 & 19 & 1 & -20 \\
\hline $\mathrm{Mn}$ & 6 & 0 & -5 & 11 & 0 & -7 & 9 & 1 & -5 & 4 & -3 & -3 \\
\hline $\mathrm{Fe}$ & 7 & 0 & -7 & 17 & -0 & -17 & 24 & -1 & -24 & 28 & -0 & -27 \\
\hline $\mathrm{Co}$ & 5 & 1 & -2 & 9 & 1 & 9 & 29 & -6 & 22 & 34 & -0 & 26 \\
\hline $\mathrm{Ni}$ & 6 & -0 & -7 & 16 & 0 & -17 & 20 & -1 & -19 & 21 & -1 & -19 \\
\hline $\mathrm{Cu}$ & 7 & -0 & -8 & 21 & -1 & -29 & 22 & 1 & -35 & 23 & -5 & -47 \\
\hline $\mathrm{Zn}$ & 8 & 1 & -7 & 18 & 1 & -21 & 22 & -2 & -29 & 29 & 1 & -47 \\
\hline $\mathrm{Sr}$ & 6 & 0 & -3 & 7 & -1 & 0 & 11 & -2 & 1 & 13 & -3 & -3 \\
\hline $\mathrm{Y}$ & 6 & -0 & -6 & 16 & -1 & -15 & 26 & 1 & -17 & 25 & 9 & -15 \\
\hline $\mathrm{Zr}$ & 3 & -0 & -3 & 6 & -3 & -5 & 8 & 0 & -5 & 11 & 2 & 2 \\
\hline $\mathrm{Ba}$ & 7 & 0 & -7 & 18 & 1 & -19 & 5 & -1 & -16 & 8 & -1 & -13 \\
\hline $\mathrm{La}$ & 7 & 1 & -5 & -5 & -3 & 3 & -12 & -0 & 7 & -22 & -2 & 22 \\
\hline $\mathrm{Ce}$ & 8 & 0 & -7 & 17 & 1 & -8 & 22 & 1 & -20 & 19 & -5 & -38 \\
\hline $\mathrm{Nd}$ & 7 & 0 & -8 & 12 & 1 & 1 & 1 & 1 & 4 & -1 & 1 & 12 \\
\hline $\mathrm{Sm}$ & 10 & 1 & -10 & -2 & -2 & -2 & -14 & 4 & 3 & -20 & -1 & 28 \\
\hline $\mathrm{Eu}$ & 3 & -1 & -5 & 1 & 1 & 1 & 10 & -1 & -14 & 17 & 14 & 5 \\
\hline
\end{tabular}

\section{Discussion}

\subsection{Rotational velocities}

\subsubsection{Comparison with other works}

Figure 6 illustrates the comparison of our determinations of $V \sin i$ with works of Uesugi \& Fukuda (1982), Abt \& Morell (1995), and Royer et al. (2002). Our values are slightly larger than those of Abt \& Morell, especially for $V \sin i$ larger than $100 \mathrm{~km} \mathrm{~s}^{-1}$. However, the trend is in the other direction when comparing with Uesugi \& Fukuda. Finally, comparison with Royer et al. is excellent if we neglect one point ( $\sim 150 \mathrm{~km} \mathrm{~s}^{-1}$ for our estimate). This point corresponds to HD 12311. This star is still classified as a dwarf in Simbad even though it has the atmospheric parameters of a giant. This was already noted by Hauck (1986) using GENEva photometry, and Gray \& Garrison (1989b) give a spectral type of F0 III-IVn. Royer et al. (2002) use Fourier transforms of several line profiles to estimate $V \sin i$. This technique is very efficient for stars with well separated lines. In cooler stars, it becomes harder to find isolated lines to analyze. HD 12311 is precisely one of the coolest stars of Royer et al. (2002). In this case, all lines of their list except one were unusable. Therefore the difference can be explained by a poor precision of their determination for this star. In this comparison, there are less points because Royer et al. focus on A type stars.

We conclude that our determination of $V \sin i$ are probably even better than those of Royer et al., because we make use of all lines present in the spectra, whether they are blended or not. On the other hand, we neglect macroturbulence, so our $V \sin i$ values are slightly biased towards large values in very slow rotators $\left(V \sin i<8-10 \mathrm{~km} \mathrm{~s}^{-1}\right)$.

\subsubsection{Systematic abundance error due to rotation}

When dealing with large rotational velocities, one can easily underestimate the continuum level. That leads to underestimated abundances because lines seem shallower.

The apparent abundances of three elements are strongly correlated with $V \sin i$. These elements are $\mathrm{Sr}, \mathrm{Sc}$, and $\mathrm{Na}$. This is illustrated in Fig. 7, where iron was also included to show the behavior of an uncorrelated element. The correlations involving $\mathrm{Sr}, \mathrm{Sc}$ and $\mathrm{Na}$ are spurious, however, for the following reasons:

Strontium: this element shows the most significant correlation (correlation coefficient $r=-0.61$ ). The problem arises 

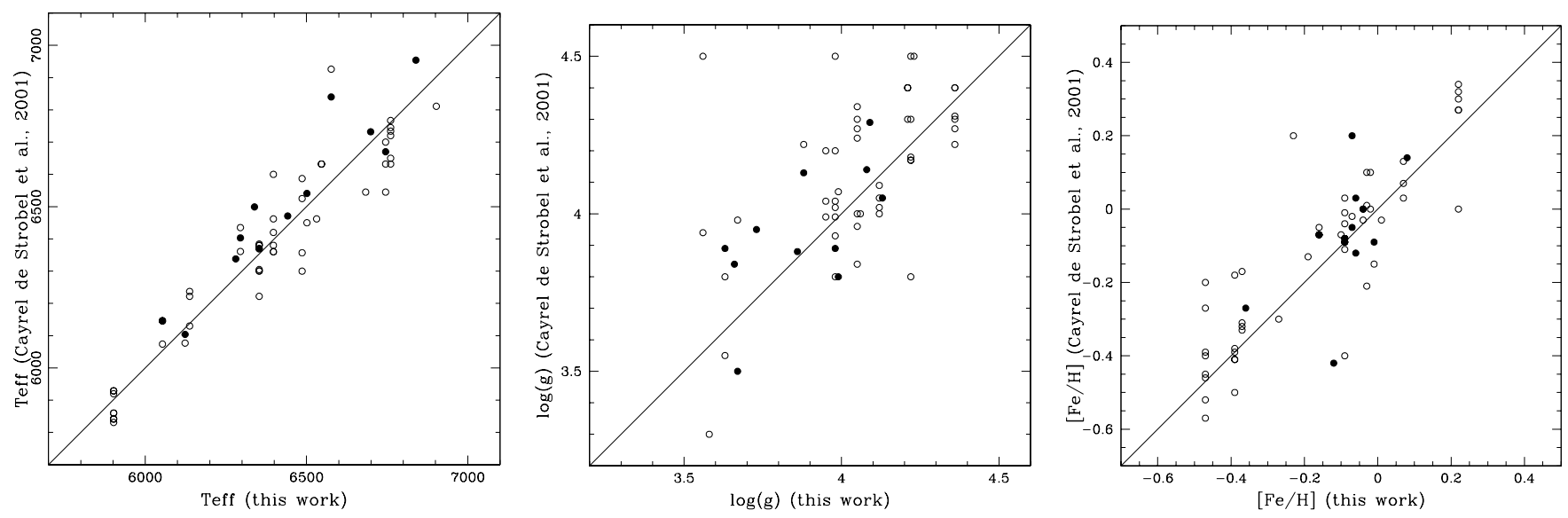

Fig. 4. Comparison between $T_{\text {eff }}, \log g,\left[\frac{\mathrm{Fe}}{\mathrm{H}}\right]$ from this work and from the $\left[\frac{\mathrm{Fe}}{\mathrm{H}}\right]$ catalogue of Cayrel de Strobel et al. (2001). Filled circles distinguish Balachandran's (1990) contribution, open circles representing determinations by other authors. The straight line is not an adjustment but represents the equality of both values.

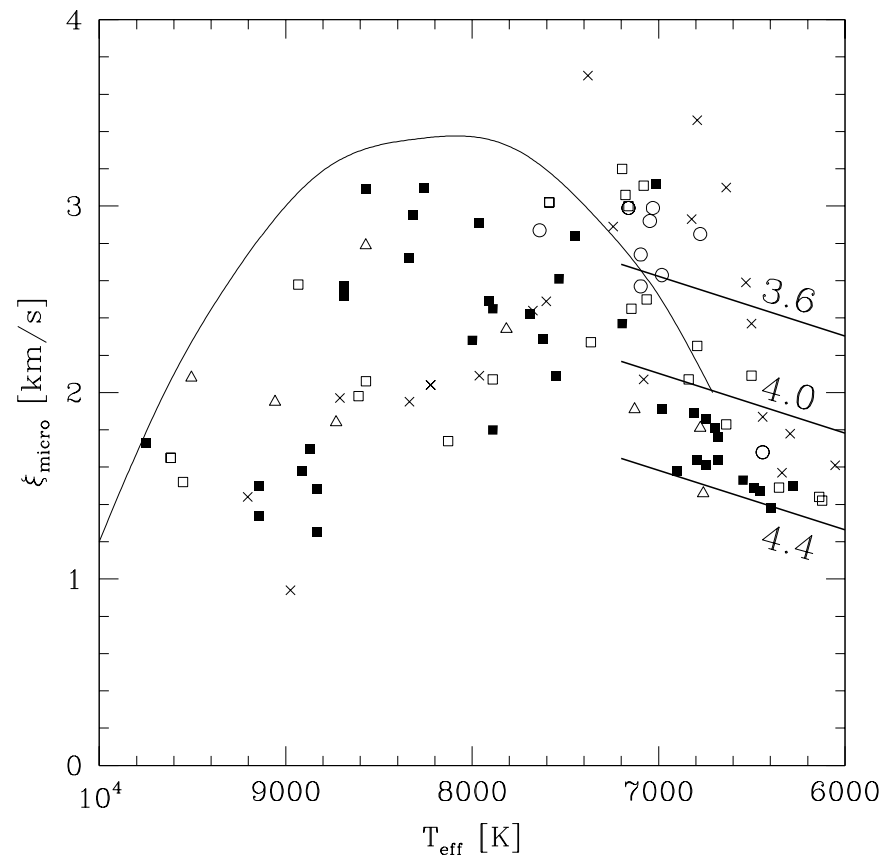

Fig. 5. Relation between $T_{\text {eff }}$ and $\xi_{\text {micro }}$ for stars with a reliable $\xi_{\text {micro }}$ determination (see text): $\triangle$ stars with $\log g>4.25$. $\mathbf{~ : ~ s t a r s ~ w i t h ~} 4.0<$ $\log g \leq 4.25$. $\square$ : stars with $3.75<\log g \leq 4.0$. $\times$ : stars with $3.5<$ $\log g \leq 3.75$. $\bigcirc$ : stars with $\log g \leq 3.5$. The continuous line is the relation of Coupry \& Burkhart (1992), while the thick straight lines are Nissen's (1981) relation for the three indicated $\log g$ values.

when only one line, Sr II $\lambda 4215.519$, is visible. This happens mainly in the hotter stars of the sample. The continuum is slightly underestimated in this region. When rotational velocity increases, this small error leads to large underestimates of the abundances. As there is no other line of $\mathrm{Sr}$ for those stars, this error cannot be corrected. In Fig. 7, almost all stars with $V \sin i>150 \mathrm{~km} \mathrm{~s}^{-1}$ are of spectral type A, which reflects the well-known drop of rotational velocity for spectral types $\mathrm{F}$ and later. It is for these relatively hot stars that only one Sr II line is used, and in this case, the result is much poorer than in the simulation illustrated by Fig. 3, which involves an F star where two Sr lines are used.

This is the only negative correlation for all adjusted elements. It is very interesting to illustrate the accuracy of the continuum adjustment, and the stability of this method. Indeed, an underestimate of the continuum leads to a negative correlation. The fact that only one element shows such a behaviour is a good justification for our choice of continuum adjustment.

Scandium: this element yields the largest positive correlation $(r=0.5)$. It should be remembered that during the analysis, spectra are cut into 7 parts, each part is adjusted, and finally the different estimates are put together (see Paper I for details). In the case of Sc, there is a large scatter between abundances of different spectral ranges. Moreover, since Sc is ionized, the adjustment is very sensitive to gravity (see Sect. 5.1). However, there is no correlation between $\log g$ and $V \sin i$. Unfortunately, the reason of this behaviour was not found, but it is probable that there are several causes adding up.

Sodium: the last significant correlation $(r=0.43)$ is found for $\mathrm{Na}$. For this element, the reason was found to be the telluric lines. The Na I doublet ( $\lambda 5889$ and $\lambda$ 5895) lies within telluric lines. Even if the adjustment is not very sensitive to these lines, a small error can lead to a large abundance overestimate, especially for fast rotators, because these lines are broad and saturated.

There is no significant correlation between other elements and $V \sin i$.

\subsubsection{Correlation between rotation and true abundances}

The decrease of $\left[\frac{\mathrm{Fe}}{\mathrm{H}}\right]$ with increasing $V \sin i$ found by Takeda \& Sadakane (1997) and confirmed by Varenne \& Monier (1999) is 

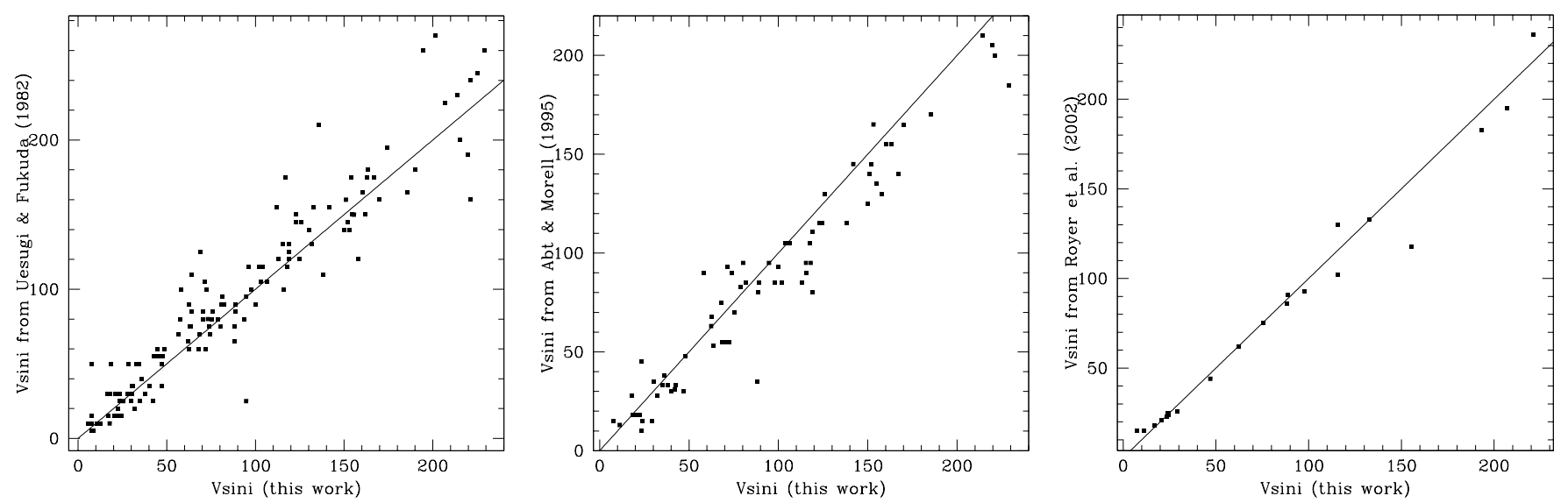

Fig. 6. Comparison between $V \sin i$ values from this work and Uesugi \& Fukuda (1982), Abt \& Morell (1995) and Royer et al. (2002). The straight line is not an adjustment but represents the equality of both values.

Table 3. Characteristics of the observed metallic giants.

\begin{tabular}{ccccc}
\hline \hline HD & $T_{\text {eff }}$ & $\log g$ & $V \sin i$ & spectral type \\
\hline 432 & 6792 & 3.59 & 74.47 & F2 III \\
12311 & 7079 & 3.67 & 155.54 & F0 V \\
15233 & 6698 & 3.81 & 130.54 & F2 III \\
79940 & 6367 & 3.42 & 118.82 & F5 III \\
115604 & 7046 & 3.27 & 7.61 & F3 III \\
147547 & 7063 & 3.23 & 151.77 & A9 III \\
155203 & 6516 & 3.65 & 161.86 & F2 V (Cr) \\
157919 & 6576 & 3.67 & 46.96 & F3 III \\
159876 & 7244 & 3.65 & 23.41 & F0 IIIb ((Sr)) \\
172748 & 6776 & 3.47 & 25.51 & F2 III* \\
181333 & 7161 & 3.41 & 47.83 & F0 III \\
186155 & 6637 & 3.70 & 44.76 & F5 II-III \\
196524 & 6441 & 3.50 & 46.10 & F6 III \\
200723 & 6591 & 3.57 & 93.42 & F2 III \\
205852 & 6934 & 3.38 & 163.43 & F3 III(a) \\
208741 & 6683 & 3.82 & 36.94 & F3-5 III \\
214441 & 6823 & 3.53 & 122.73 & F1 III \\
214470 & 6637 & 3.59 & 81.00 & F3 III-IV \\
\hline
\end{tabular}

firmly denied by our results. Figure 8 shows clearly that there is no dependence of iron or nickel abundance with $V \sin i$. In our case, there are 60 stars with $V \sin i \geq 100 \mathrm{~km} \mathrm{~s}^{-1}$ while there was only 10 in Varenne \& Monier and 5 in Takeda and Sadakane. It is important to notice that the anticorrelation was found for stars of the Hyades, but, a priori, there is no reason why only these stars should be affected. The result of Takeda et al. (1997) is discussed in more detail in Sect. 7.3

\subsection{Metallic giants}

Figure 9 shows the position of all giant stars of our sample in the HR diagram. There are 35 giants, out of which 18 are metallic. Their characteristics are reported in Tables 3 and 4.

Abundances of observed giants are illustrated in Fig. 10. There are no very clear-cut peculiarities such as the underabundance of $\mathrm{Ca}$ and/or Sc in Am stars. However, abundances
Table 4. Characteristics of the observed normal giants.

\begin{tabular}{ccccc}
\hline \hline HD & $T_{\text {eff }}$ & $\log g$ & $V \sin i$ & spectral type \\
\hline 1671 & 6441 & 3.66 & 44.20 & F5 III \\
2628 & 7161 & 3.78 & 20.84 & A7 III \\
12573 & 7961 & 3.72 & 118.09 & A5 III \\
118216 & 6531 & 3.69 & 16.82 & F5 III \\
127762 & 7585 & 3.74 & 123.02 & A7 III \\
142357 & 6501 & 3.58 & 30.11 & F5 II-III \\
150557 & 6854 & 3.93 & 63.26 & F2 III \\
151769 & 6295 & 3.63 & 12.48 & F7 III \\
159561 & 7798 & 3.89 & 214.04 & A5 III \\
166230 & 7638 & 3.46 & 42.54 & A8 III \\
176303 & 6053 & 3.56 & 27.69 & F8 III \\
181623 & 6729 & 3.55 & 112.03 & F2 III \\
182900 & 6338 & 3.73 & 31.08 & F6 III \\
186005 & 6870 & 3.69 & 151.02 & F1 III \\
187764 & 6982 & 3.33 & 102.00 & F0 III \\
197051 & 7638 & 3.53 & 75.68 & A7 III \\
208450 & 7095 & 3.49 & 131.66 & F0 III-IVn \\
\hline
\end{tabular}

of some elements (Al, Ca, Ti, Fe, Ba for example) are clearly different.

To ensure that the abundances are really different, the Kolmogorov-Smirnov test was used. This test gives the probability that two distributions are drawn from the same parent population.

It was already shown in North et al. (1998) that the $\log g$ distribution of metallic and normal giants are different with a probability of $99.99 \%$. However, the $T_{\text {eff }}$ distributions are comparable. The distributions of $V \sin i$ and $\xi_{\text {micro }}$ estimated by our method are also comparable.

Figure 11 shows the cumulative distribution of abundances for each of the 6 elements that have the largest differences. They are $\mathrm{Ca}, \mathrm{Ti}, \mathrm{Mn}, \mathrm{Fe}, \mathrm{Ni}$ and $\mathrm{Ba}$. $\mathrm{Al}$ and $\mathrm{Cr}$ also have different distributions. Sc is not added, even though the corresponding distributions are clearly different, because of its correlation with $V \sin i$. 

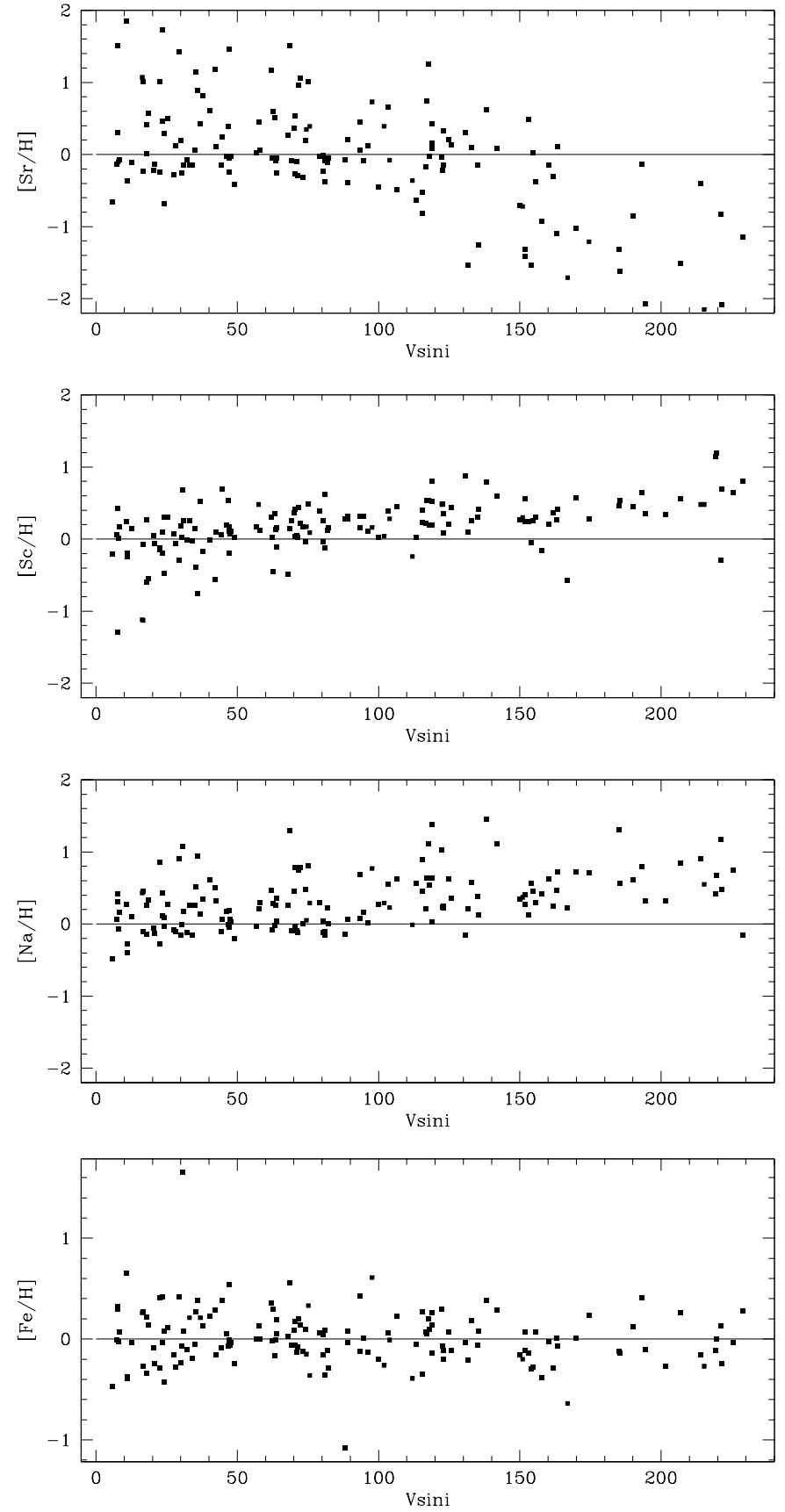

Fig. 7. Behaviour of $\mathrm{Sr}, \mathrm{Sc}, \mathrm{Na}, \mathrm{Fe}$ with respect to $V \sin i$.

Thus, we conclude that metallic giants have abundances of $\mathrm{Al}, \mathrm{Ca}, \mathrm{Ti}, \mathrm{Cr}, \mathrm{Mn}, \mathrm{Fe}, \mathrm{Ni}$ and $\mathrm{Ba}$ that are significantly larger than those of normal giants.

\subsection{Abundance variation with evolution}

Recently, the Montréal group has published a new study for normal stars. Models including all effects of atomic diffusion and radiative accelerations have been presented for stars of different masses in Turcotte et al. (1998), Richer et al. (2000) and Richard et al. (2001).

The models of Turcotte et al. predict the evolution of the abundances for an F star of a given mass consistently with the internal structure. They are computed in the diffusion framework with only the mixing length as free-parameter. They include the effect of 28 chemical elements $(Z \leq 28)$ but no macroscopic mixing. Atomic diffusion plays an important role for stars more massive than $1.3 \mathrm{M}_{\odot}$. We agree with Varenne \& Monier (1999) that the theoretical predictions of Turcotte et al. (1998) for F stars are not confirmed by observations. In our case, abundance of $\mathrm{C}, \mathrm{O}, \mathrm{Si}, \mathrm{Ca}, \mathrm{Fe}$, and $\mathrm{Ni}$ do not show any variations with effective temperature (see Fig. 12).

Richard et al. (2001) present stellar models that are evolved from the pre-main sequence to the giant branch for stars of 1.3 to $4.0 M_{\odot}$ and metallicities of $Z=0.01$ to 0.03 . In order to compare our observations with these models, our sample was split into 7 mass groups with masses below $1.4 M_{\odot}, 1.4$ to $1.6 M_{\odot}, 1.6$ to $1.8 M_{\odot}, \ldots, 2.4$ to $2.6 M_{\odot}$ and above $2.6 M_{\odot}$. Figure 13 shows the behaviour of $\mathrm{Ca}, \mathrm{Fe}$ and $\mathrm{Ni}$ for stars of 1.6 to $1.8 M_{\odot}$ and 1.8 to $2.0 M_{\odot}$ with respect to $\log g$. It can be directly compared with Fig. 10 of Richard et al. (2001). They predict a large abundance variation of at least 1 dex with decreasing $\log g$ (or equivalently, with time). In our case, there is no variation with $\log g$ except for stars of 1.8 to $2.0 M_{\odot}$, and even in this case, the amplitude is much smaller than the theoretical predictions. In order to confirm the statistical significance of the correlation, $2 \times 2$ contingency tables have been computed, segregating stars with gravities larger or lower than $\log g=4.0$ and with $[\mathrm{Fe} / \mathrm{H}]$ or $[\mathrm{Ni} / \mathrm{H}]$ larger or lower than 0.0 . The corresponding $\chi^{2}$ were computed taking Yates' correction into account (Crow et al. 1960) and the results were 8.98 and 10.94 for $[\mathrm{Fe} / \mathrm{H}]$ and $[\mathrm{Ni} / \mathrm{H}]$ respectively, which comfortably exceeds the 7.88 value corresponding to a $0.5 \%$ probability that abundances and $\log g$ are unrelated. Thus, taken at face value, the correlation is statistically significant. If this variation is physically true, it suggests that additional hydrodynamical processes are at play in these stars, which lower the effects of microscopic diffusion. However, before concluding to the physical reality of this correlation, it is necessary to test how far this behaviour can be explained by a correlation of abundances with $V \sin i$ or by NLTE effect.

Takeda \& Sadakane (1997) also found a variation of iron abundance with $\log g$, which was qualitatively the same, for Hyades' members. They explain this variation as a superficial phenomenon due to the existence of a mutual relationship between $\log g$ and $V \sin i$. They found an anticorrelation between $V \sin i$ and $\log g$, in the sense that high-gravity stars tend to be slow rotators. They consider that the cause of this relation is rotation, by which the apparent and effective values of $\log g$ are affected. In their case, $\log g$ is estimated from Strömgren photometry, and rotation affects this determination: it appears that $\log g$ values progressively decrease with increasing $V \sin i$.

However, in our case, $\log g$ values were estimated from Hipparcos data, and they are not correlated with $V \sin i$ (see Fig. 14). The effect of rotation is to increase the $B 2-V 1$ index. Maeder (1971) has shown that, to a good approximation, this index is essentially sensitive to the product $V \sin i$. For a star of $1.4 M_{\odot}\left(2.0 M_{\odot}\right.$ respectively $)$, a $V \sin i$ of $200 \mathrm{~km} \mathrm{~s}^{-1}$ leads to a decrease in $\log \left(T_{\text {eff }}\right)$ of 0.016 ( 0.010 respectively). The effect on $M_{V}$ is of a few hundredths of a magnitude $(\sim 0.1$ mag respectively) (Maeder \& Peytremann 1970). This affects slightly the 

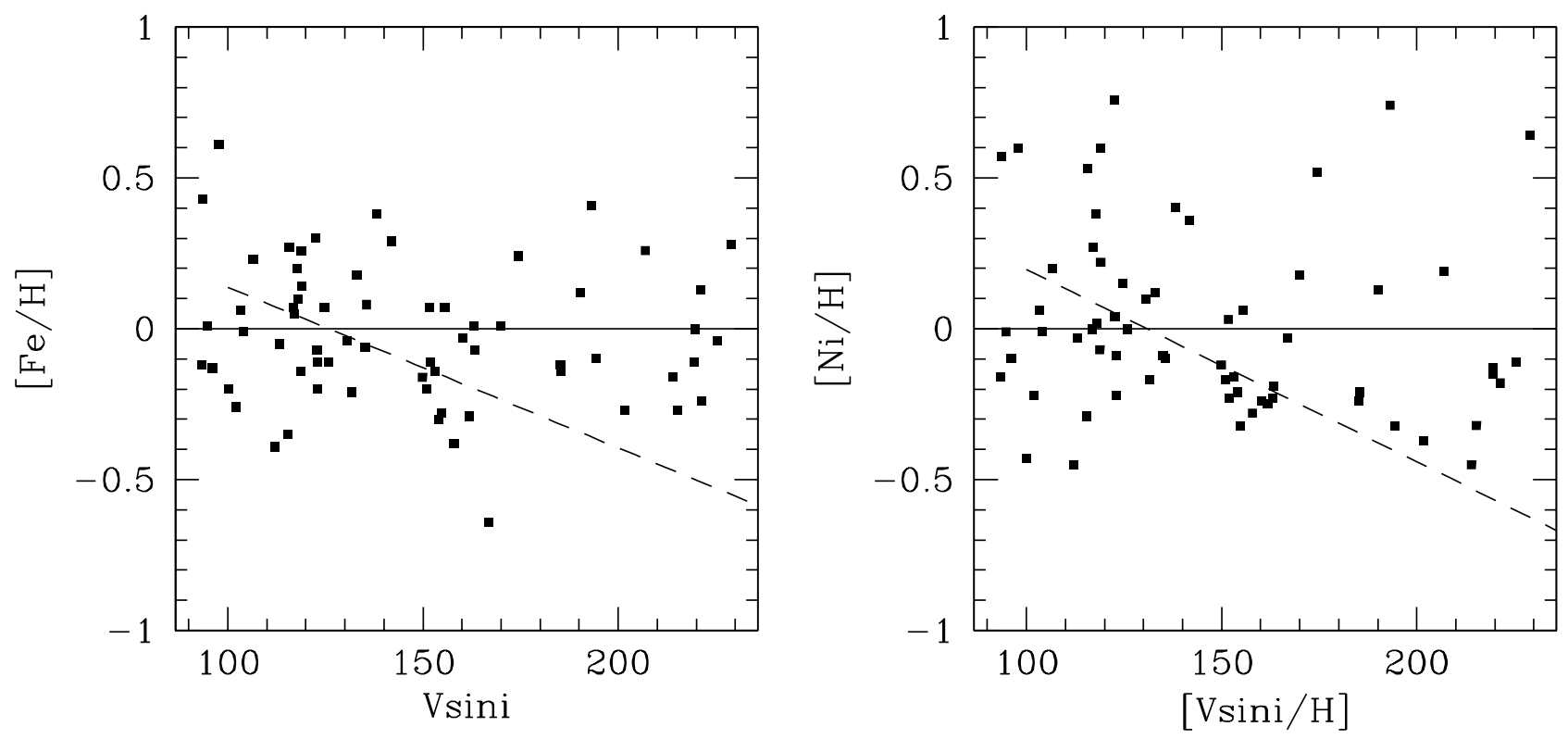

Fig. 8. Abundances of $\mathrm{Fe}$ and $\mathrm{Ni}$ as a function of $V \sin i$ for fast rotators. The dashed line represent the anticorrelation proposed by Varenne \& Monier (1999).

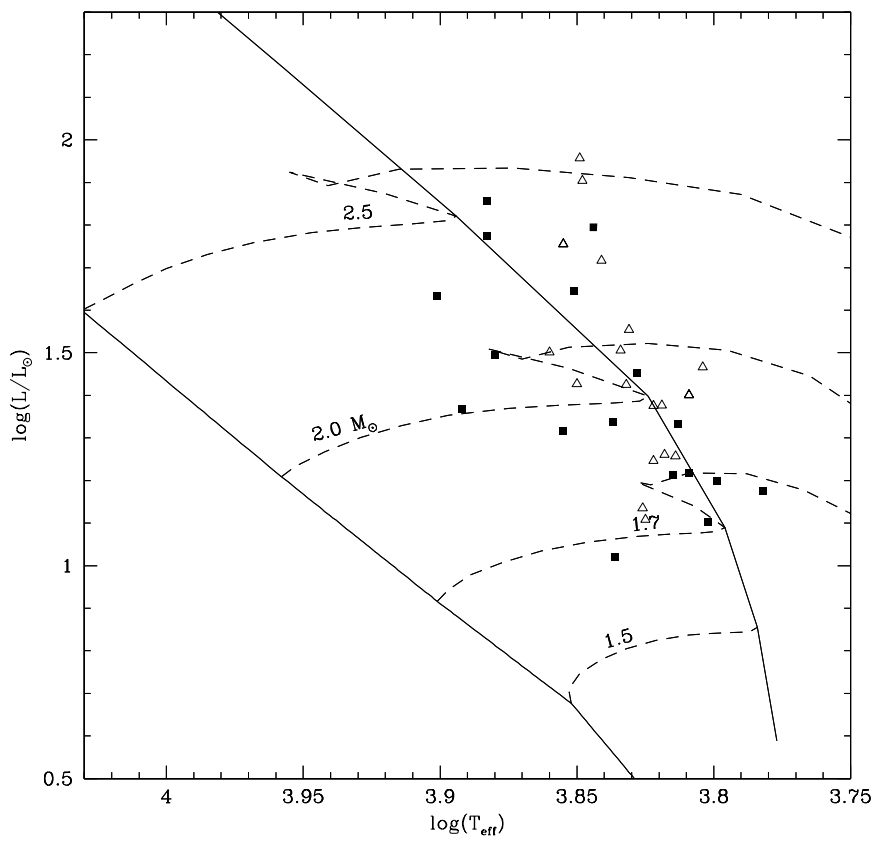

Fig. 9. HR diagram of giant stars with some some evolutionary tracks

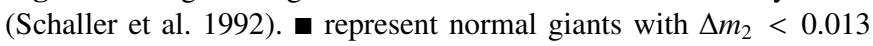
and $\Delta$ represent metallic giants with $\Delta m_{2} \geq 0.013$.

mass estimate when interpolating through evolutionary tracks of non-rotating models, but the error cannot exceed $0.1 M_{\odot}(5 \%$ at $2 M_{\odot}$ ), propagating into no more than $\sim 0.02 \operatorname{dex}$ in $\log g$. In fact our estimates of $\log g$ are close to the effective gravity, i.e. the sum of gravitational and centrifugal accelerations average over the visible disk; we verified that they agree quite well with photometric estimates computed from the $u v b y \beta$ colours and the calibration by Moon \& Dworetsky (1985). The interesting thing with effective $\log g$ is that for $V \sin i \sim 200 \mathrm{~km} \mathrm{~s}^{-1}$, it differs by no more, on the equator, than 0.1 dex from a non-rotating $2 M_{\odot}$ star near the ZAMS, while the difference is at least twice as large at the turn-off. Thus the observed $\log g$ axis (e.g. in Fig. 13) is slightly stretched for fast rotators, relative to the $\log g$ non-rotating stars would have in the same mass range. But since slow rotators are not affected, the net effect is just to blur very slightly the relation metallicity vs. $\log g$ shown in Fig. 13. Therefore, this cannot explain the behaviour of $\left[\frac{\mathrm{Fe}}{\mathrm{H}}\right]$ with $\log g$. Moreover, in Takeda \& Sadakane (1997), $\log g$ varies on a range that is no more than 0.2 dex; under the assumption that this variation is due entirely to a bias in the $\log g$ estimate, it can hardly explain a 0.4 dex variation of $\left[\frac{\mathrm{Fe}}{\mathrm{H}}\right]$ for the single line of neutral iron they used.

Given the variation of $T_{\text {eff }}$ and $\log g$ for these stars, NLTE effects might play an important role. Rentzsch-Holm (1996) has estimated NLTE effects on iron abundances for A-type stars. NLTE correction increase with $T_{\text {eff }}$ going from 7000 to 10000 K (see Fig. 4 of Rentzsch-Holm 1996). On the other hand, at given effective temperature, they increase with decreasing gravity. Since both $T_{\text {eff }}$ and $\log g$ decrease with time when a star is evolving on the main sequence, this tends to flatten the NLTE correction. For our stars of 1.8 to $2.0 M_{\odot}$, there is a small NLTE correction of 0.1 to 0.15 dex. However, this correction is almost constant (variation of $0.05 \mathrm{dex}$ ) for the $T_{\text {eff }}$ and $\log g$ considered $^{3}$. Moreover, with our method, we do not have separate abundance values for Fe I and Fe II. Let us recall that Fe II lines are almost not affected by NLTE effects. When $T_{\text {eff }}$ increases, the ratio of line numbers of $\mathrm{Fe}_{\mathrm{I}}$ and $\mathrm{Fe}$ II decreases. Therefore our determination becomes less sensitive to NLTE effects. This also tends to flatten the variation of NLTE correction with $T_{\text {eff }}$. Therefore, we conclude that, for stars of 1.8 to

\footnotetext{
${ }^{3}$ Note that this is valid only if $T_{\text {eff }}$ and $\log g$ are correlated. The Pearson product-moment correlation coefficient is 0.972 (0.968) for stars of 1.8 to $2.0 M_{\odot}\left(1.6\right.$ to $1.8 M_{\odot}$ respectively), and in the same order for the other mass intervals.
} 


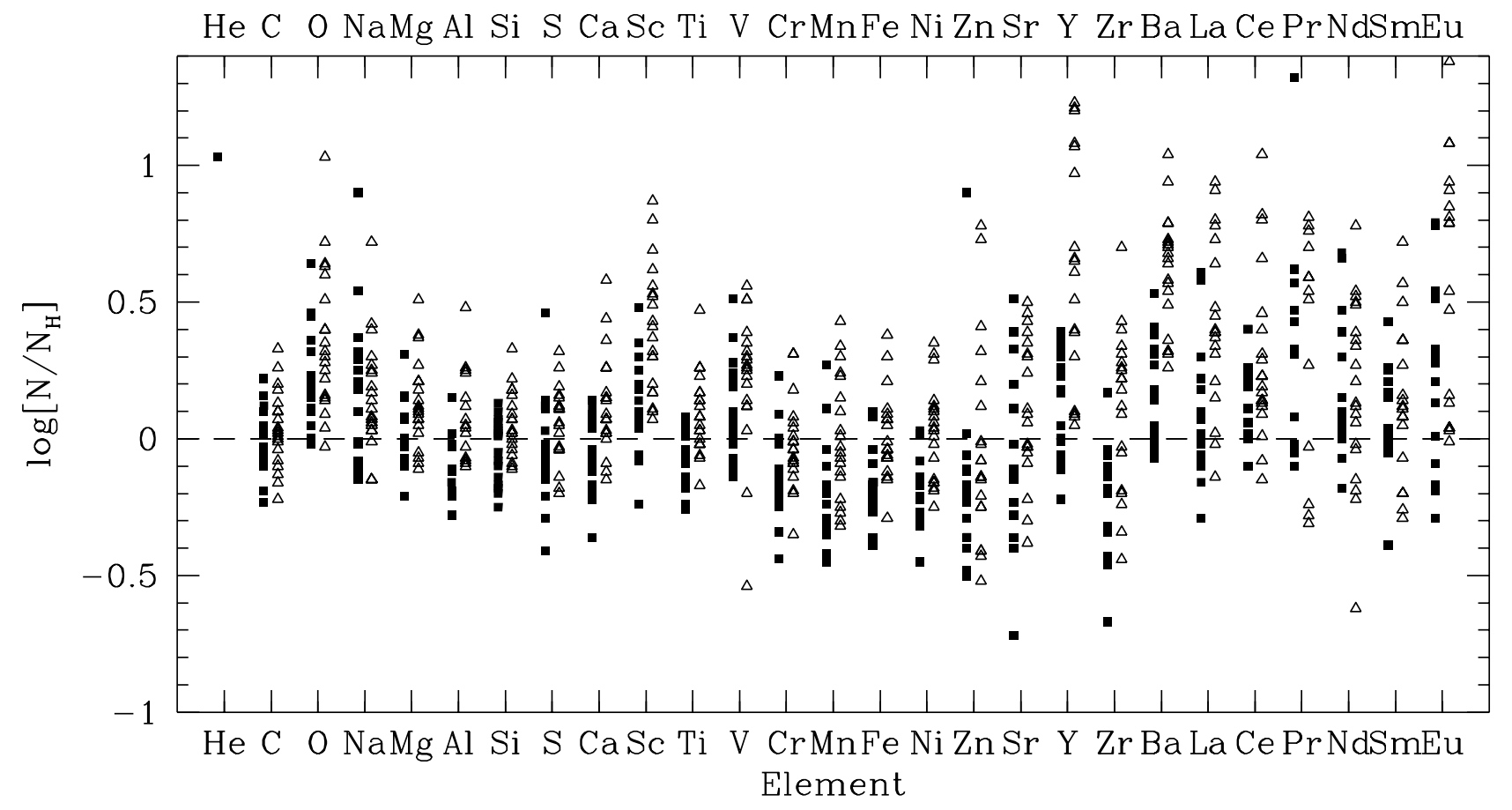

Fig. 10. Abundance pattern of normal giants $(\mathbf{\square})$ and of metallic giants $(\triangle)$.
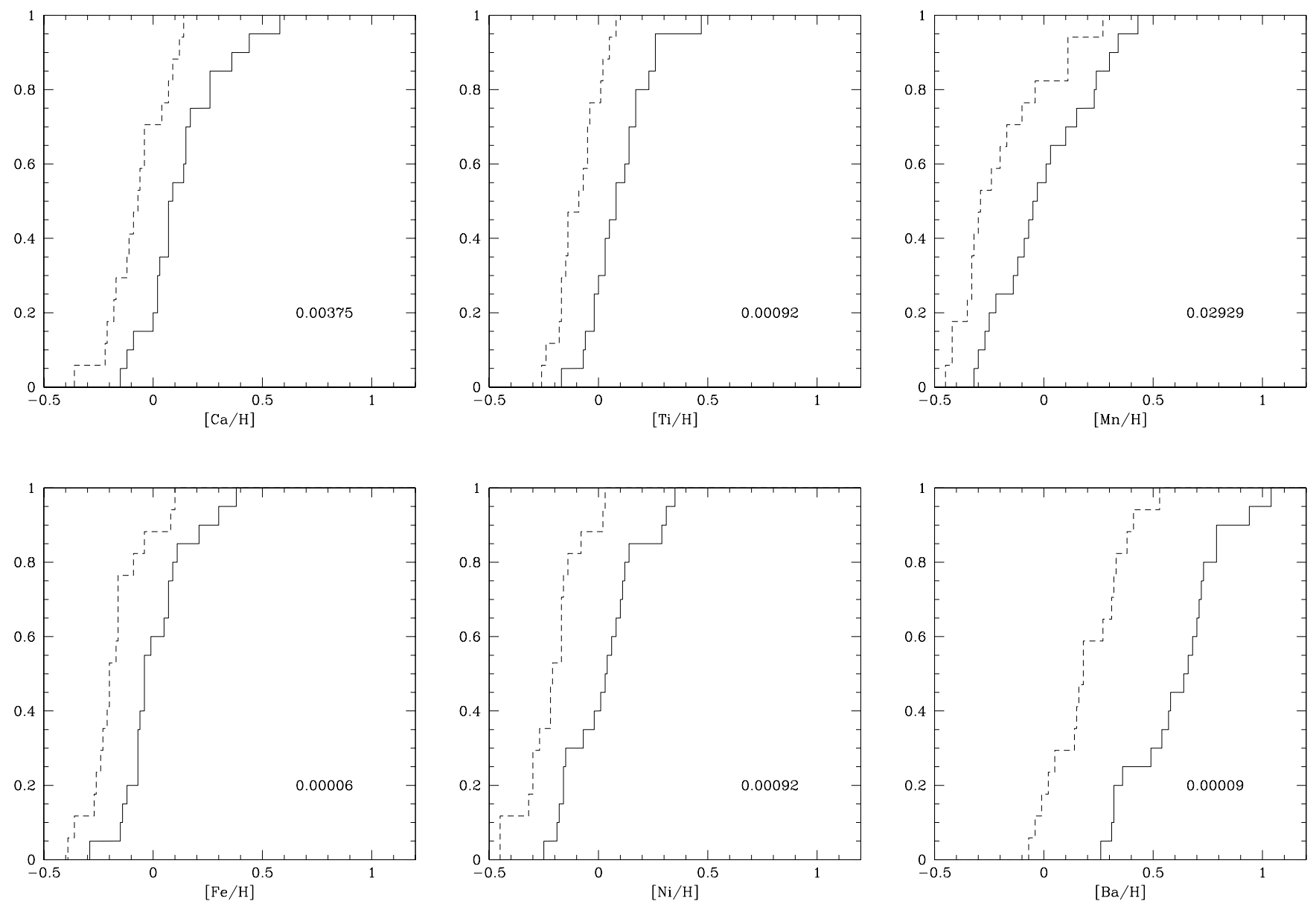

Fig. 11. Cumulative distributions of abundances of metallic giants (solid line) and of normal one (dashed line). The number in each panel indicates the probability that both distributions are drawn from the same parent distribution. 

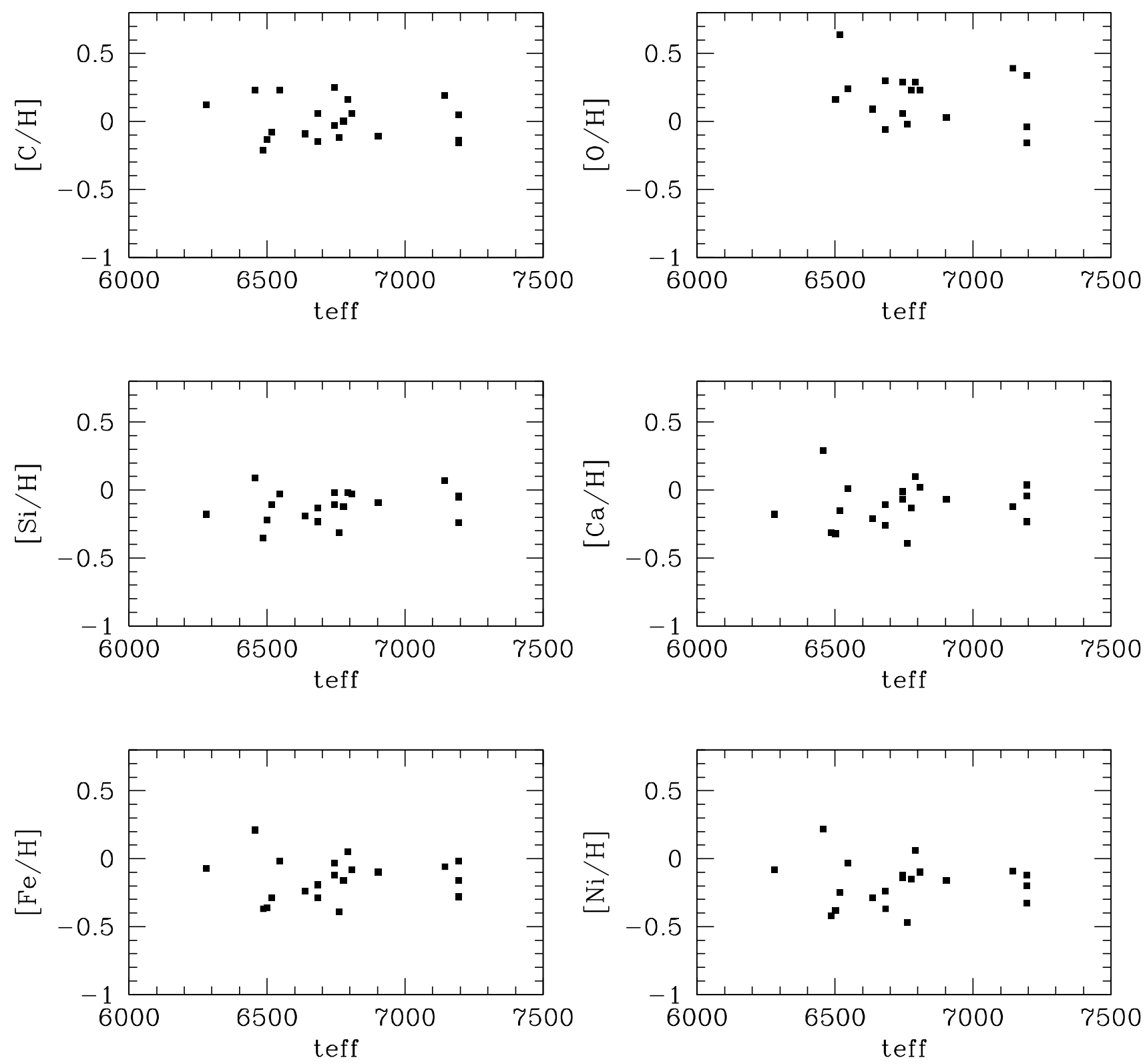

Fig. 12. Abundances of dwarf F-type stars as a function of $T_{\text {eff }}$.

2.0 $M_{\odot}$, only a small NLTE correction of about $0.1 \mathrm{dex}$, that is roughly constant with $T_{\text {eff }}$, has to be taken into account when iron abundance is estimated with our method. To summarize, all points in the $\left[\frac{\mathrm{Fe}}{\mathrm{H}}\right]$ vs. $\log g$ diagram for stars of $1.8-2.0 M_{\odot}$ in Fig. 13 should be shifted upward by about 0.1 to obtain a more realistic picture.

Thévenin \& Idiart (1999) discuss the problem of $\log g$ determination when dealing with NLTE correction. Their work focuses on cooler stars, but the problem of $\log g$ determination is comparable. When the NLTE correction is applied to LTE abundances, abundances of Fe I and Fe II are no longer the same (assuming that $\log g$ had been adjusted to satisfy the LTE ionization equilibrium). Therefore, $\log g$ has to be corrected until ionization equilibrium is satisfied after NLTE correction. Nissen et al. (1997) have shown that there exists a discrepancy between spectroscopic $\log g$ (adjusted assuming LTE) and those deduced from HIPPARcos parallaxes.
Thévenin \& Idiart (1999) have solved this discrepancy by adjusting $\log g$ after NLTE correction; the latter are in close agreement with those deduced from HipParcos (our $\log g$ are estimated in this way). However, in order to apply NLTE corrections precisely, it is necessary to have separate abundance estimates of Fe I and Fe II, which is not our case. Roughly, our determination can be seen as an average of both abundances weighted by the number of lines of each ionization stage.

To summarize, NLTE effect on iron is no more than $0.1 \mathrm{dex}$ and remains constant to better than $0.1 \mathrm{dex}$, for stars of 1.8 to $2.0 M_{\odot}$ when abundance are estimated with our method.

In view of the above discussion, it seems that the correlation between $\left[\frac{\mathrm{Fe}}{\mathrm{H}}\right]$ and $\log g$ for $1.8-2.0 M_{\odot}$ is indeed physical, and due neither to systematic errors nor to selection effects. It agrees qualitatively with the prediction of Richard et al. (2001), but it is strange that only the stars of 1.8 to $2.0 M_{\odot}$ follow their 

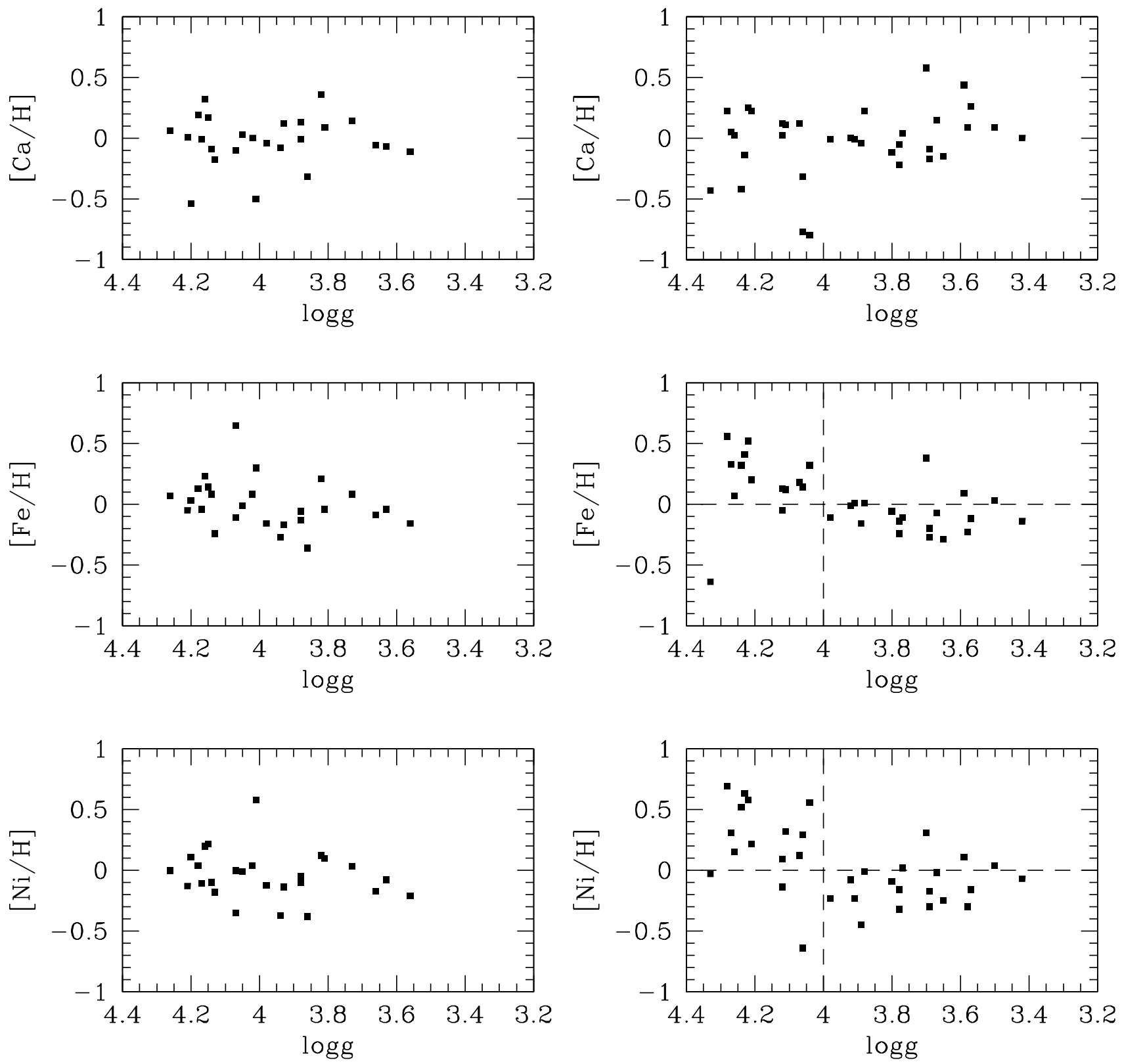

Fig. 13. Relation between abundances of $\mathrm{Ca}, \mathrm{Fe}$ and $\mathrm{Ni}$ with $\log g$. Left: stars of 1.6 to $1.8 M_{\odot}$. Right: stars of 1.8 to $2.0 M_{\odot}$. The broken lines show the limits of the $2 \times 2$ contingency tables mentioned in the text.

models. It is true that more massive stars are less numerous, so their statistics is less good. For less massive stars, models predict a variation that occurs on a shorter $\log g$ range. It is possible that this variation is too quick to be observed, but then we would expect to see at least an increase of the scatter for large $\log g$ values, while this is not the case.

The comparison with the Montréal models shows that it is necessary to add hydrodynamical processes in order to reproduce observations, like mass loss and meridional circulation, as already suggested by the Montréal group. Maybe turbulence will also have to be modeled in a slightly different way, yet to be defined.

We also note that the metal-rich 1.8 to $2.0 M_{\odot}$ stars lie just around the blue border of the instability strip or outside of it: the coolest of these (HD 144197) has $T_{\text {eff }}=7691 \mathrm{~K}$ and the

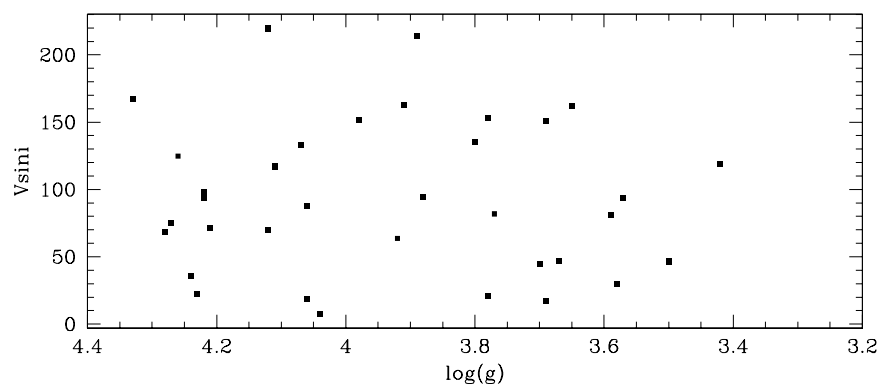

Fig. 14. Plot showing $V \sin i$ as a function of $\log g$ for stars of 1.8 to $2.0 M_{\odot}$.

next cooler one (HD 148367) has $T_{\text {eff }}=7962 \mathrm{~K}$, while the blue limit of the instability strip is defined by $B 2-V 1=-0.01$ 
on the ZAMS (Jasniewicz 1984) which correspond to $T_{\mathrm{eff}}=$ $8016 \mathrm{~K}$ according to the calibration of Hauck \& Künzli (1996). The hottest metal-rich star (HD 195943) has $T_{\text {eff }}=9057 \mathrm{~K}$. Almost all stars with $[\mathrm{Fe} / \mathrm{H}]<0.0$ are cooler and lie, therefore, either inside the instability strip or to the red side of it. This might be more than a coincidence, and is reminiscent of the well known exclusion between $\delta$ Scuti type pulsations and the Am phenomenon. The difference is that while most Am stars do lie inside the instability strip even though they do not pulsate, the metal-rich 1.8 to $2.0 M_{\odot}$ stars are found either outside or on the verge of it.

\section{Conclusion}

The method of analysis presented in Paper I was used to derive detailed abundances of $140 \mathrm{~A}$ and F-type stars with $V \sin i$ up to about $200 \mathrm{~km} \mathrm{~s}^{-1}$. $V \sin i$ derived with this method are consistent with those in the literature. Only $\mathrm{Cr}, \mathrm{Sc}$, and $\mathrm{Na}$ are very sensitive to rotational velocity in the sense that their abundance determination is altered when $V \sin i \geq 100 \mathrm{~km} \mathrm{~s}^{-1}$, for various reasons.

This study allows us to specify more exactly the nature of metallic A-F giants. They are more evolved than normal giants and they show overabundances of at least 8 elements with respect to normal giants. These elements are $\mathrm{Al}, \mathrm{Ca}, \mathrm{Ti}, \mathrm{Cr}, \mathrm{Mn}$, $\mathrm{Fe}, \mathrm{Ni}$ and $\mathrm{Ba}$.

Our results do not confirm, in general, the predictions of the Montréal models. However, stars of 1.8 to $2.0 M_{\odot}$ show a clear correlation of iron abundances with $\log g$ which is reminiscent of theoretical predictions by Richard et al. (2001). Neither rotational velocity nor NLTE effects can explain this behaviour, which appears, therefore, to be real. The observed $\left[\frac{\mathrm{Fe}}{\mathrm{H}}\right]$ and $\left[\frac{\mathrm{Ni}}{\mathrm{H}}\right]$ variations qualitatively agree with those predicted by the Montréal models, but are much weaker. The smaller amplitude indicates that their models need more hydrodynamical processes (meridional circulation, mass loss, ... ) and/or a better tuning of convection. Our large homogeneous set of abundances hopefully can help this work.

Acknowledgements. We express our warm thanks to P. Bartholdi and C. Briner for their help in using the computer network of the Geneva observatory and for the instructive discussions. Constructive criticism by the referee, Dr. Richard Gray, is gratefully acknowledged. This work has been partly supported by the Swiss National Science Foundation.

\section{References}

Abt, H. A., \& Morell, N. I. 1995, ApJS, 99, 135

Balachandran, S. 1990, ApJ, 354, 310

Baranne, A., Queloz, D., Mayor, M., et al. 1996, A\&AS, 119, 373

Berthet, S. 1992, A\&A, 253, 451

Berthet, S. 1991, A\&A, 251, 171

Berthet, S. 1990, A\&A, 227, 156

Breger, M. 1979, PASP, 91, 5

Castelli, F., Gratton, R. G., \& Kurucz, R. L. 1996, A\&A, 318, 841

Cayrel de Strobel, G., Soubiran, C., \& Ralite, N. 2001, A\&A, 373, 159

Charbonnel, C., Meynet, G., Maeder, A., Schaller, G., \& Schaerer, D. 1993, A\&AS, 101, 415
Coupry, M. F., \& Burkhart, C. 1992, A\&AS, 95, 41

Cramer, N. 1999, New Astron. Rev., 43, 343

Crawford, D. L. 1975, AJ, 80, 955

Crawford, D. L. 1979, AJ, 84, 1858

Crow, E. L., Davis, F. A., \& Maxfield, M. W. 1960, Statistics Manual (Dover, New York)

Erspamer, D., \& North, P. 2002, A\&A, 383, 227 (Paper I)

ESA 1997, The HIPPARcos and Tycho Catalogues, ESA SP-1200

Flower, P. J. 1996, ApJ, 469, 355

Golay, M. 1980, Vistas Astron., 24, 141

Gray, R. O., Graham, P. W., \& Hoyt, S. R. 2001, ApJ, 121, 2159

Gray, R. O., \& Garrison, R. F. 1989a, ApJS, 70, 623

Gray, R. O., \& Garrison, R. F. 1989b, ApJS, 69, 301

Gray, R. O., \& Garrison, R. F. 1987, ApJS, 65, 581G

Hauck, B. 1986, A\&A, 155, 371

Hauck, B. 1973, in IAU Symp., 54, 117

Hauck, B., \& Künzli, M. 1996, Baltic Astron., 5, 303

Hoffleit, D., \& Jaschek, C. 1991, The bright star catalogue, 5th revised edition (Yale Univ. Obs., New Haven)

Jasniewicz, G. 1984, A\&A, 141, 116

Künzli, M., \& North, P. 1998, A\&AS, 127, 277

Künzli, M., North, P., Kurucz, R.L., \& Nicolet, B. 1997, A\&AS, 122, 51

Kurucz, R. L. 1993, ATLAS9 Stellar Atmosphere Programs and $2 \mathrm{~km} \mathrm{~s}^{-1}$ grid

Kurucz, R. L., Furenlid, I., Brault, J., \& Testerman, L. 1984, The solar flux atlas from 296 to $1300 \mathrm{~nm}$, National Solar Observatory Atlas No. 1, Sunspot, New Mexico

Maeder, A. 1971, A\&A, 10, 354

Maeder, A., \& Peytremann, E. 1970, A\&A, 7, 120

Michaud, G. 1991, in Evolution of Stars: the photospheric abundance connection, ed. G. Michaud, \& A. Tutukov (Kluwer), IAU Symp., 145, 111

Michaud,G., Tarasick, D., Charland, Y., \& Pelletier, C. 1983, ApJ, 269, 239

Moon, T. T., \& Dworetsky, M. M. 1985, MNRAS, 217, 305

Nissen, P. E. 1981, A\&A, 97, 145

Nissen, P. E., Høg, E., \& Schuster, W. J. 1997, in Hipparcos Venice '97, ESA SP-402, 225

North, P., Erspamer, D., \& Künzli, M. 1998, contributions of the Astronomical Observatory Skalnate Pleso, 27, 252

North, P., Jaschek, C., \& Egret, D. 1997, in Hipparcos - Venice '97, ESA SP-402, 367

Queloz, D., Mayor, M., Udry, S., et al. 2001, The Messenger, 105, 1

Queloz, D., Mayor, M., Weber, L., et al. 1999, A\&A, 354, 99

Rentzsch-Holm, I. 1996, A\&A, 312, 966

Richard, O., Michaud, G., \& Richer, J. 2001, ApJ, 558, 377

Richer, J., Michaud, G., \& Turcotte, S. 2000, ApJ, 529, 338

Royer, F., Gerbaldi, M., Faraggiana, R., \& Gomez, A. E. 2002, A\&A, 381,105

Rufener, F., \& Nicolet, B. 1988, A\&A, 206, 357

Schaller, G., Schaerer, D., Meynet, G., \& Maeder, A. 1992, A\&AS, 96, 269

Schaerer, D., Meynet, G., Maeder, A., \& Schaller, G. 1993a, A\&AS, 98, 523

Schaerer, D., Charbonnel, C., Meynet, G., Maeder, A., \& Schaller, G. 1993b, A\&AS, 102, 339

Takeda, Y., \& Sadakane, K. 1997, PASJ, 49, 367

Thévenin, F., \& Idiart, T. P. 1999, ApJ, 521, 753

Turcotte, S., Richer, J., \& Michaud, G. 1998, ApJ, 504, 559

Uesugi, A., \& Fukuda, I. 1982, Catalogue of stellar rotational velocities (revised), Kyoto: University of Kyoto, Department of Astronomy, Rev. Ed.

Varenne, O., \& Monier, R. 1999, A\&A, 351, 247 